\title{
SOCS2 Controls Proliferation and Stemness of Hematopoietic Cells under Stress Conditions and Its Deregulation Marks Unfavorable Acute Leukemias
}

\author{
Caterina Vitali', Claudia Bassani ${ }^{1}$, Claudia Chiodoni', Elisa Fellini ${ }^{1}$, Carla Guarnotta ${ }^{2}$, \\ Silvia Miotti ${ }^{1}$, Sabina Sangaletti ${ }^{1}$, Fabio Fuligni ${ }^{3}$, Loris De Cecco ${ }^{4}$, Pier P. Piccaluga ${ }^{3}$, \\ Mario P. Colombo ${ }^{1}$, and Claudio Tripodo ${ }^{2}$
}

\section{Abstract}

Hematopoietic stem cells (HSC) promptly adapt hematopoiesis to stress conditions, such as infection and cancer, replenishing bone marrow-derived circulating populations, while preserving the stem cell reservoir. SOCS2, a feedback inhibitor of JAK-STAT pathways, is expressed in most primitive HSC and is upregulated in response to STAT5-inducing cytokines. We demonstrate that Socs2 deficiency unleashes HSC proliferation in vitro, sustaining STAT5 phosphorylation in response to IL3, thrombopoietin, and GM-CSF. In vivo, SOCS2 deficiency leads to unrestricted myelopoietic response to 5 -fluorouracil (5-FU) and, in turn, induces exhaustion of long-term HSC function along serial bone marrow transplantations. The emerging role of SOCS2 in HSC under stress conditions prompted the investigation of malignant hematopoiesis. High levels of SOCS2 characterize unfavorable subsets of acute myeloid and lymphoblastic leukemias, such as those with
$M L L$ and $B C R / A B L$ abnormalities, and correlate with the enrichment of genes belonging to hematopoietic and leukemic stemness signatures. In this setting, SOCS2 and its correlated genes are part of regulatory networks fronted by IKZF1/Ikaros and MEF2C, two transcriptional regulators involved in normal and leukemic hematopoiesis that have never been linked to SOCS2. Accordingly, a comparison of murine $w t$ and Socs $2^{-1-}$ HSC gene expression in response to 5-FU revealed a significant overlap with the molecular programs that correlate with SOCS2 expression in leukemias, particularly with the oncogenic pathways and with the IKZF1/Ikaros and MEF2C-predicted targets. Lentiviral gene transduction of murine hematopoietic precursors with Mef2c, but not with Ikzf1, induces Socs 2 upregulation, unveiling a direct control exerted by Mef2c over Socs 2 expression. Cancer Res; 75 (11); 2387-99. ()2015 AACR

\section{Introduction}

Hematopoiesis is a regulated process in which bone marrow multipotent hematopoietic stem cells (HSC) are driven toward self-renewal or differentiation (1). At the steady state, long-term HSC (LT-HSC) remain mostly quiescent and rarely divide to produce short-term HSC (ST-HSC), multipotent precursor (MPP), and committed progenitors (2), ensuring a continuous

${ }^{1}$ Molecular Immunology Unit, Department of Experimental Oncology and Molecular Medicine, Fondazione IRCCS Istituto Nazionale de Tumori, Milan, Italy. ${ }^{2}$ Department of Human Pathology, University of Palermo, Palermo, Italy. ${ }^{3}$ Hematopathology Section, Department of Hematology and Oncology, S. Orsola-Malpighi Hospital, University of Bologna, Bologna, Italy. ${ }^{4}$ Functional Genomics Core Facility, Fondazione IRCCS Istituto Nazionale dei Tumori, Milan, Italy.

Note: Supplementary data for this article are available at Cancer Research Online (http://cancerres.aacrjournals.org/)

M.P. Colombo and C. Tripodo share senior authorship of this article.

Corresponding Author: Mario P. Colombo, Molecular Immunology Unit, Department of Experimental Oncology and Molecular Medicine, Fondazione IRCCS Istituto Nazionale dei Tumori, Via Amadeo 42, 20133 Milan, Italy. Phone: 39-0223902252; Fax: 39-02-23903073; E-mail:

mariopaolo.colombo@istitutotumori.mi.it

doi: 10.1158/0008-5472.CAN-14-3625

(C)2015 American Association for Cancer Research. flow of differentiated hematopoietic cells. Under hematopoietic stress, such as infections, traumata, myeloablation, and cancer, HSC on one side proliferate to reconstitute the affected hematopoietic pools or expand specific hematopoietic populations such as myeloid-derived suppressor cells in the presence of cancer; on the other side, even in conditions of emergency hematopoiesis, they do need to maintain the stem cell reservoir $(3,4)$.

HSC quiescence, activation, self-renewal, and differentiation under bone marrow stress are regulated by cytokines (5), including stem cell factor (SCF), IL3, thrombopoietin (TPO), erythropoietin (EPO), Flt3/Flk2 ligand (Flt3L), GM-CSF, G-CSF, and IL6. Signal transduction of most hematopoietic cytokines passes through the Janus-activated kinase (JAK) and signal transducer and activator of transcription (STAT; ref. 5). Suppressor of cytokine signaling (SOCS) family comprises eight members (SOCS17 and CIS) with similar structures, which are induced upon JAK/ STAT activation and function as negative feedback. SOCS1 and SOCS3 immunologic roles are widely recognized $(6,7)$. SOCS2 has been initially described as a regulator of the growth hormoneinsulin-like growth factor 1 axis $(8,9)$ and only recently has been recognized as controller of immunologic functions (10-12). SOCS2 basal expression is higher in HSC (particularly LT-HSC) than in differentiated populations (13-16), and it is upregulated following STAT5 activation by hematopoietic cytokine stimulation (17) or following myeloablative 5-fluorouracil (5-FU) treatment (18). Induction of Socs2 by G-CSF, IL3, GM-CSF, and EPO 
has been documented in cell lines and tissues, including the bone marrow (19).

In hematologic malignancies (20-22), the JAK-STAT pathways are frequently deregulated $(10,11)$, with oncogenic activation of STAT3 and STAT5 in acute myeloid leukemia (AML), and of STAT1 and STAT5 in pre-B acute lymphoblastic leukemia (B-ALL) and chronic myelogenous leukemia (CML; ref. 20), with potential involvement of SOCS2.

Here, we have uncovered a novel role for SOCS2 in the regulation of HSC functions in settings of bone marrow myelopoietic stress response and, by thorough analysis of SOCS2 expression in human hematopoietic malignancies, we have identified SOCS2 deregulation as part of a stemness-related molecular signature characterizing unfavorable acute myeloid and lymphoblastic leukemia subsets.

\section{Materials and Methods}

Mice

Socs $2^{-1-}$ mice were obtained from Dr. C. Farquharson [The Roslin Institute and Royal (Dick) School of Veterinary Studies, University of Edinburgh, Roslin, United Kingdom] and backcrossed to C57BL/6NCrl for 10 generations. Six- to 8-week-old age- and sex-matched wild-type $(w t)$ and $\operatorname{Socs} 2^{-1-}$ littermates were used for experiments. C57BL/6NCrl mice were purchased from Charles River. CD45.1 mice were bred in our animal facility. Experiments were performed according to local ethical guidelines.

\section{5-FU myeloablation}

Mice were injected intraperitoneally with $150 \mathrm{mg} / \mathrm{kg}$ body weight of 5-FU (TEVA). White blood cells (WBC) were counted on blood samples collected in heparinized tubes and counts were expressed as $\mathrm{WBC} / \mu \mathrm{L}$ of blood.

\section{Cell preparation and bone marrow assays}

Bone marrow cells were flushed from tibias and femurs and spleens were mechanically disrupted in PBS and passed through $70-\mu \mathrm{m}$ cell strainers (BD). Erythrocytes were lysed and remaining cells were resuspended in PBS. For purification of bone marrow lineage-negative cells $\left(\mathrm{Lin}^{-}\right)$, we used mouse lineage cell depletion kit (Miltenyi Biotec) following the manufacturer's instructions. Details about serial bone marrow transplantation (BMT) experiments, in vitro colony-forming cell assay, in vitro cytokine stimulation, FACS, and phospho-STAT5 staining are included in the Supplementary Methods.

\section{Histopathology and IHC}

Histopathologic analyses on mouse tissues were performed on sections stained with hematoxylin and eosin. IHC on human bone marrow was performed as previously described (23) using a specific anti-human SOCS2 antibody (Abcam). Detailed description of the analyzed human bone marrow samples is provided in the Supplementary Methods.

\section{Human public dataset analysis}

For computational gene expression profiles (GEP) analyses, public human leukemia datasets were used. Detailed information about samples and methods used for analysis are included in the Supplementary Data.

\section{Microarray}

For gene expression experiment, $w t$ and Socs $2^{-1-} \mathrm{Lin}^{-} \mathrm{CKit}^{+} \mathrm{Sca}-$ $1^{+}$(LKS) from 6 mice/group were individually sorted in TRIzol as described in the Supplementary Data. Bead Chip Array MouseWG-6 v2 (Illumina) was used for transcriptome analysis. Detailed procedures used for RNA extraction, cDNA amplification, microarray, data preprocessing, and analysis are included in the Supplementary Data. Gene expression data are deposited in NCBI's Gene Expression Omnibus database (accession number GSE66065).

Lentiviral production and infection of Lin ${ }^{-}$cells

The pReceiver-Lv215 lentiviral vectors containing the fulllength open reading frame (ORF) CDNA of mouse Mef $2 c$ (NM_001170537) and Ikzf1 (NM_001025597) or the empty vector were purchased from GeneCopoeia. A third-generation packaging system involving the transfection of four plasmids in the producer cells, namely two packaging plasmids (pMDLg/ pRRE and pRSV-REV), an envelope plasmid (pMD2-VSV-G), and the lentiviral transfer vector, was used. Lentiviral stocks were produced in $293 \mathrm{~T}$ cells by $\mathrm{Ca}_{3} \mathrm{PO}_{4}$ cotransfection of the four plasmids. Supernatants were collected, passed through a $0.22-\mu \mathrm{m}$ filter, and purified by ultracentrifugation as described previously (22). Lin ${ }^{-}$cells isolated from $w t$ mice were prestimulated overnight in Stemspan SFEM (STEMCELL Technologies) supplemented with $100 \mathrm{ng} / \mathrm{mL}$ SCF, $100 \mathrm{ng} / \mathrm{mL}$ Flt $3 \mathrm{~L}, 50 \mathrm{ng} / \mathrm{mL}$ TPO, $20 \mathrm{ng} / \mathrm{mL}$ IL3, and then lentiviral particles were added at a multiplicity of infection (MOI) of 100. After 24 hours, cells were washed and cultured without the cytokine cocktail; for positive control, untransduced cells were left in the presence of cytokines; 48 hours later, cells were collected and lysed in TRIzol and used for subsequent analysis.

\section{Statistical analysis}

Data in the histograms of Figs. 1-4 and 6 were represented as mean $\pm \mathrm{SEM}$, and an unpaired two-tailed Student $t$ test was routinely calculated otherwise specified in the figure legends.

\section{Results}

Hematopoietic stress responses are deregulated in SOCS2deficient mice

Expression of Socs 2 in HSC under basal conditions was high in LKS multipotent HSC and declined in lineage-committed common myeloid progenitors (CMP), granulocyte/monocyte progenitors (GMP), and megakaryocyte/erythroid progenitors (MEP Supplementary Fig. S1A and S1B), consistent with Socs2 characterizing the stem cell compartment $(13-16,22,25)$. Still, SOCS2 was dispensable for basal hematopoiesis being the bone marrow composition of $w t$ and Socs $2^{-1-}$ mice similar at the steady state (Supplementary Fig. S1C and S1D). We hypothesized that SOCS2, as inhibitor of JAK-STAT signaling, could influence HSC functions in settings of hematopoietic stress. Therefore, we studied the bone marrow response to myeloablation induced by 5 -FU in $w t$ and Socs $2^{-1-}$ mice. After 5-FU, a wave of cytokines is produced and induces bone marrow regeneration, which occurs around day $10(3,21)$. Socs 2 was rapidly induced in bone marrow Lin $^{-}$cells (a population enriched for HSC) 1 day after 5-FU administration and reached 30-fold upregulation after 6 days of treatment, returning to baseline within day 10 (Fig. 1A). Following 5-FU treatment, WBC counts similarly reached nadir within 4 days, but Socs $2^{-1-}$ mice fully recovered between day 7 and 9 , while $w t$ mice recovered at day 11 , a time point at which Socs $2^{-1-}$ WBC counts even exceeded baseline (Fig. 1B). At day 14 , Socs $2^{-/-}$mice 
A

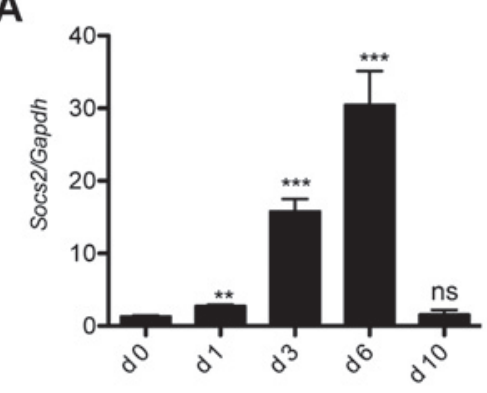

D

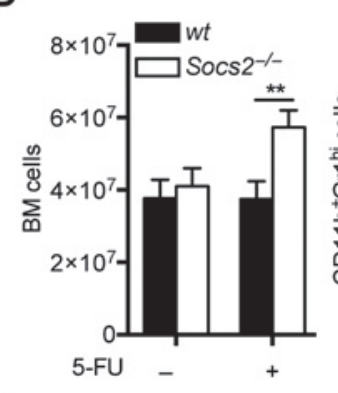

B

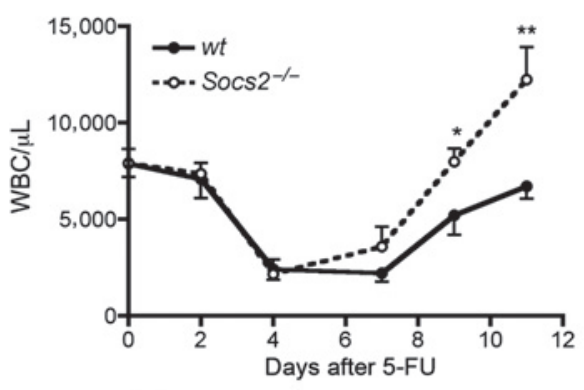

C

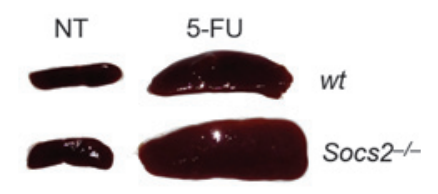

E

F
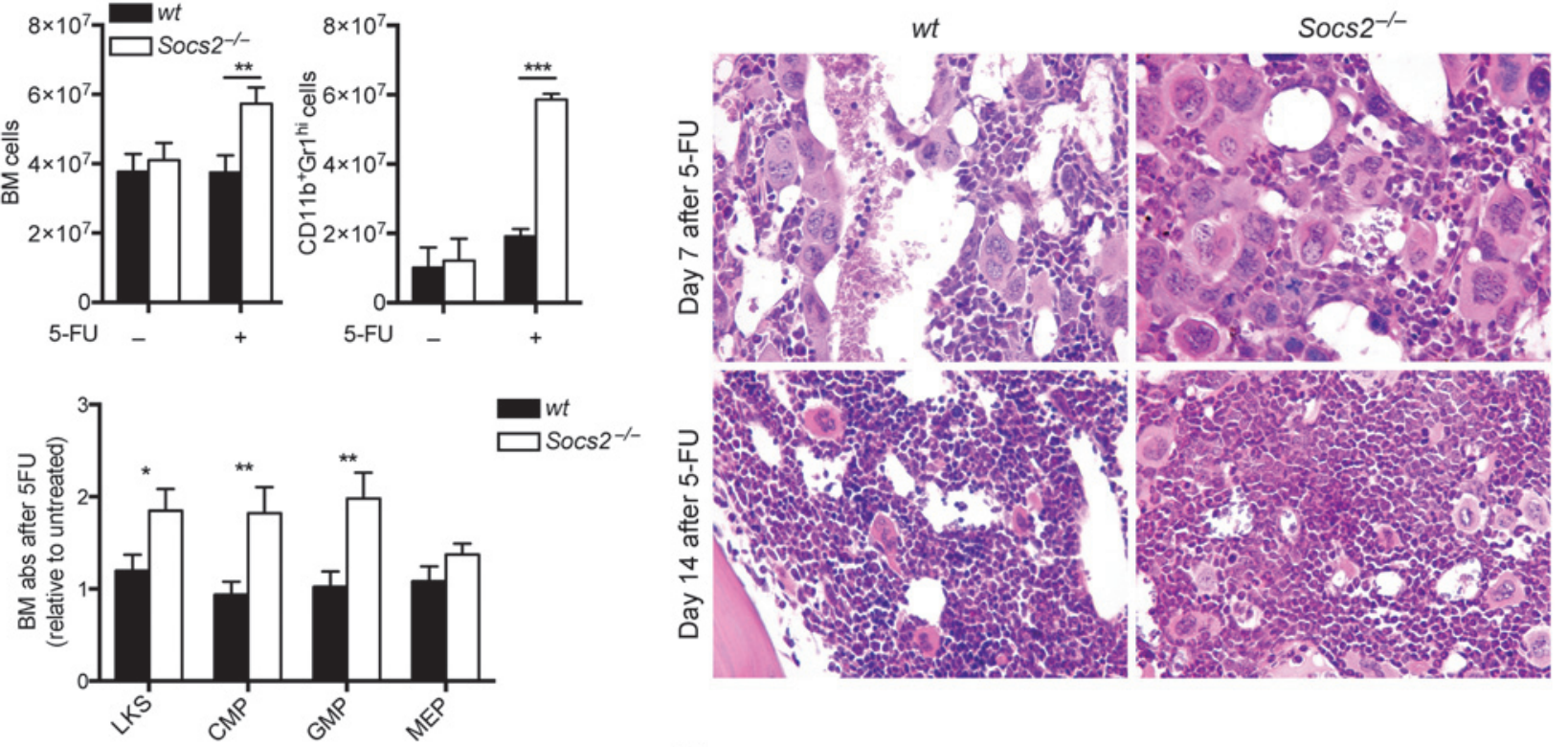

G
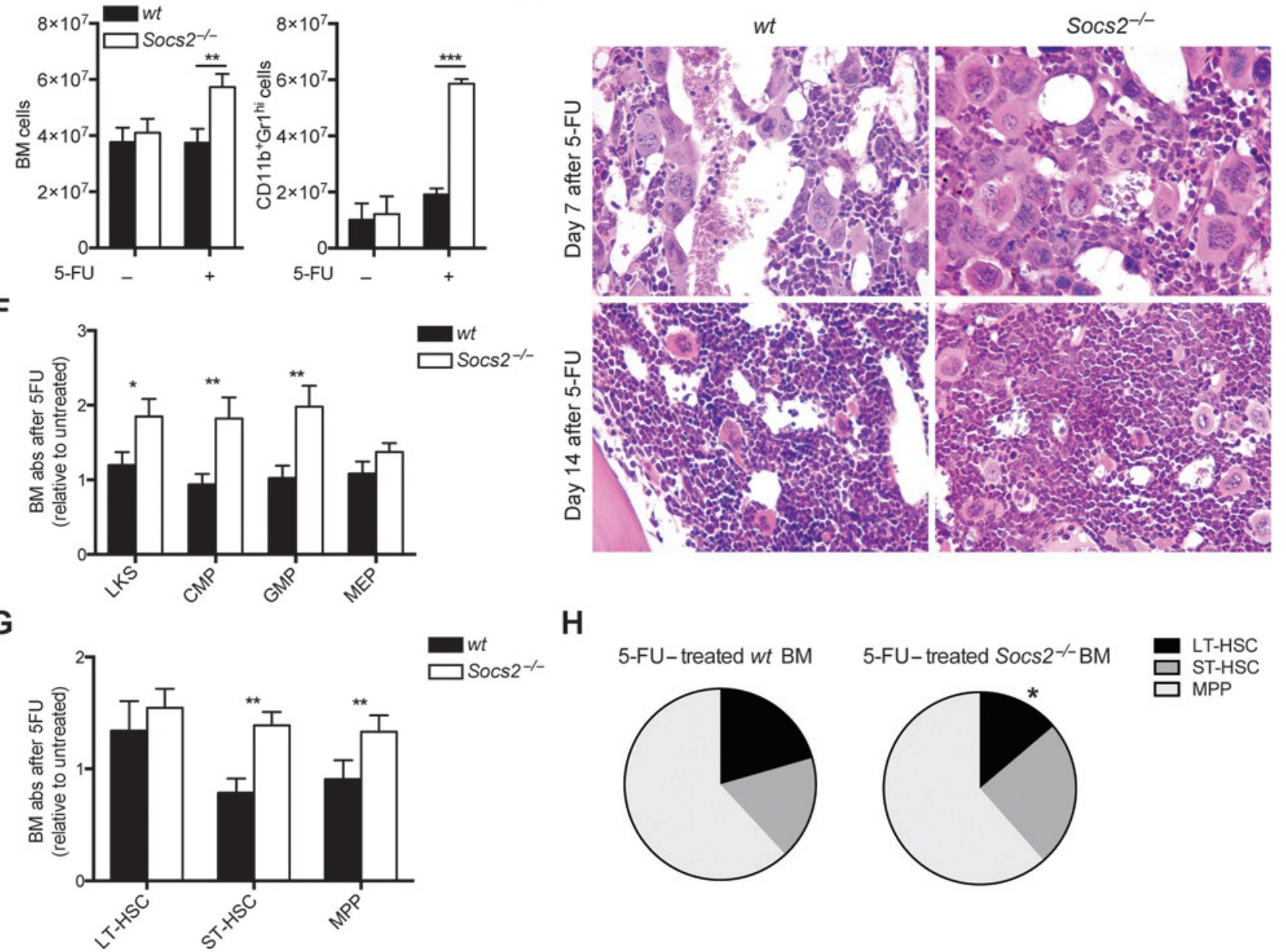

H
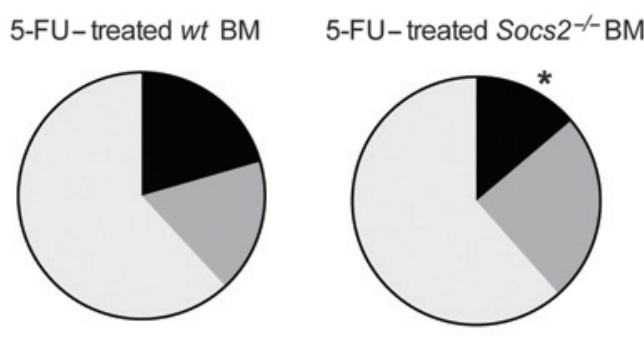

Figure 1.

SOCS2 limits HSC response to 5-FU. A, qPCR of Socs 2 in bone marrow Lin $^{-}$cells from mice at the indicated time points showed transient increase of Socs 2 soon after 5-FU treatment (5 mice/each time point). B, WBC counts following 5-FU treatment showed accelerated and unrestrained rebound in Socs $2^{-/-}$compared $^{-14}$ with $w t$ mice. C, splenomegaly occurred in Socs $2^{-1-}$ mice after 5-FU. D, whole bone marrow and $\mathrm{CD}_{11 \mathrm{~b}}{ }^{\mathrm{G}} \mathrm{Gr} 1^{+}$cell counts from one femur 14 days after 5-FU revealed the overwhelming expansion of bone marrow cells in Socs $2^{-1-}$ mice. E, histologic analysis of bone marrow sections of mice 7 and 14 days after 5 -FU treatment showed increased expansion of megakaryocytic and granulocytic elements in Socs $2^{-/-}$mice. F and G, absolute cell numbers of the indicated bone marrow populations in mice treated with 5-FU indicated that expansion of committed progenitors was evident in Socs $2^{-1-}$ mice upon treatment. Results are shown as ratio of counts from treated animals compared with the mean absolute number of three untreated mice of the same strain. H, relative frequency of LT-HSC $\left(\mathrm{CD} 150^{+} \mathrm{CD} 48^{-}\right)$, ST-HSC $\left(\mathrm{CD} 150^{+} \mathrm{CD} 48^{+}\right)$, and MPP $\left(\mathrm{CD}_{150^{-}} \mathrm{CD} 48^{+}\right)$showed increased frequency of committed progenitors at the expense of LT-HSC. Results of $5-$ FU experiments are from three independent experiments ( $n=7$ treated mice and $n=3$ untreated mice/group).

displayed splenomegaly (Fig. 1C) and increased bone marrow cellularity (Fig. 1D) especially in the granulocytic compartment, suggesting an unrestrained myelopoietic response. Histologic analysis confirmed greater expansion of granulocytes and mega- karyocytes in Socs $2^{-/-}$than in $w t$ mice at day 7 and 14 after 5-FU (Fig. 1E).

The excessive expansion of differentiated hematopoietic populations in response to 5-FU treatment in Socs ${ }^{-1-}$ mice was 
A

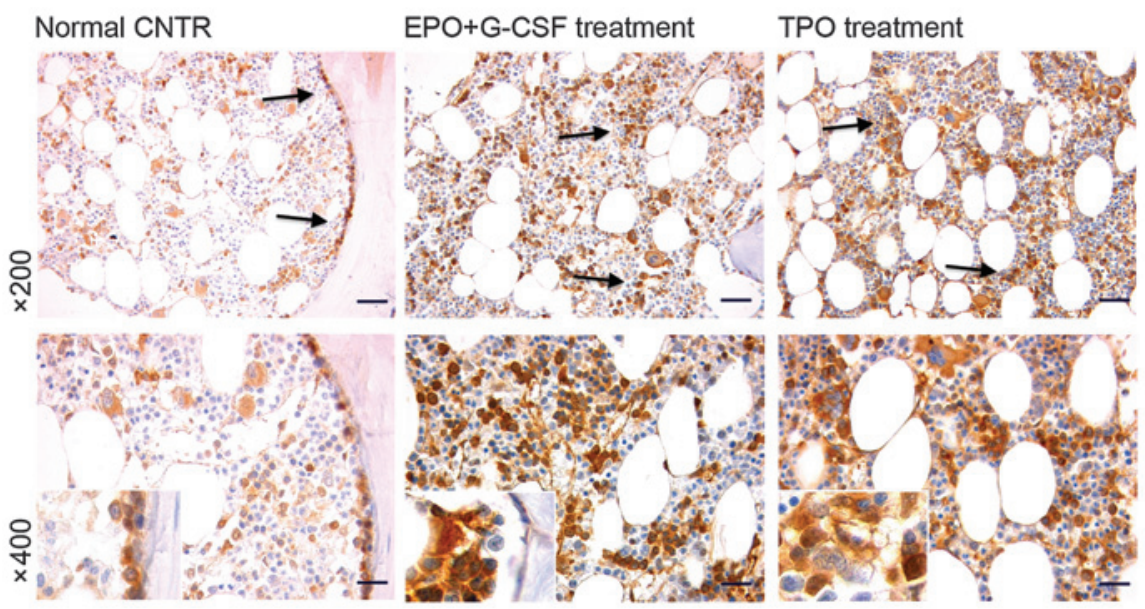

B
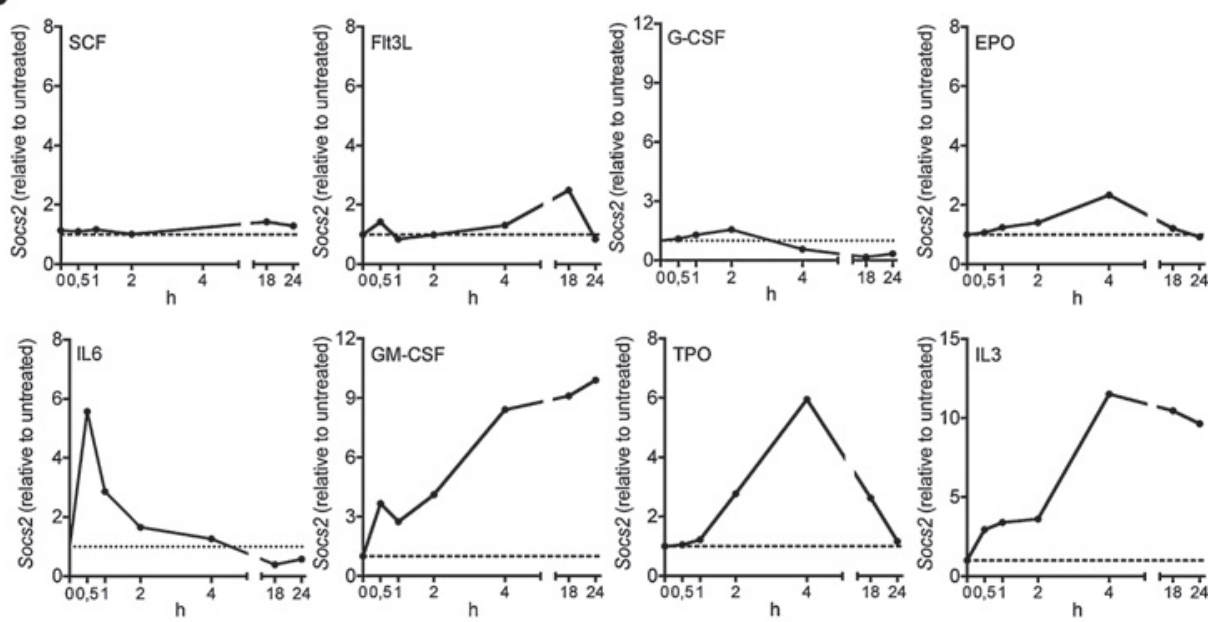

C

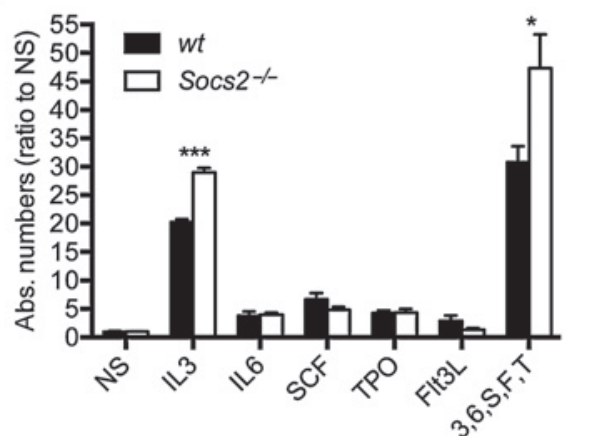

D

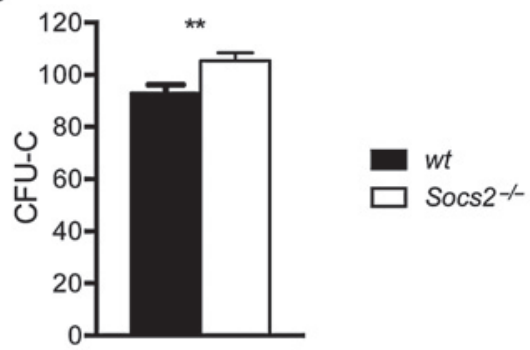

consequence of LKS, CMP, GMP, and MEP amplification (Fig. 1F), which outnumbered their $w t$ counterpart. Further characterization of LKS using SLAM markers CD48/CD150 showed that the unrestricted expansion of LKS in the absence of SOCS2 was confined to ST-HSC and MPP progenitors (Fig. 1G) with reduced LT-HSC relative frequency (Fig. $1 \mathrm{H}$ ).

SOCS2 is induced upon cytokine signaling and in turn controls cytokine-mediated HSC expansion

SOCS2 induction was observed in patients treated with hematopoietic cytokines. In normal bone marrow, SOCS2

marked hematopoietic precursors lining the bone trabeculae (Fig $2 \mathrm{~A}$, left, black arrows) and scattered immature precursors, whereas SOCS2 stained expanded erythroid, myeloid, and megakaryocytic precursor clusters (black arrows) in bone marrow from patients treated with EPO and G-CSF (Fig. 2A, middle) or TPO (Fig. 2A, right).

In mice, TPO, IL3, and GM-CSF strongly induced Socs2, whereas SCF, G-CSF, Flt3L, IL6, and EPO were negligible Socs 2 inducers in bone marrow Lin $^{-}$cells (Fig. 2B).

To explore the role of SOCS2 in HSC following cytokine stimulation, we cultivated $w t$ or Socs $2^{-1-}$ LKS for 3 days in the 
A

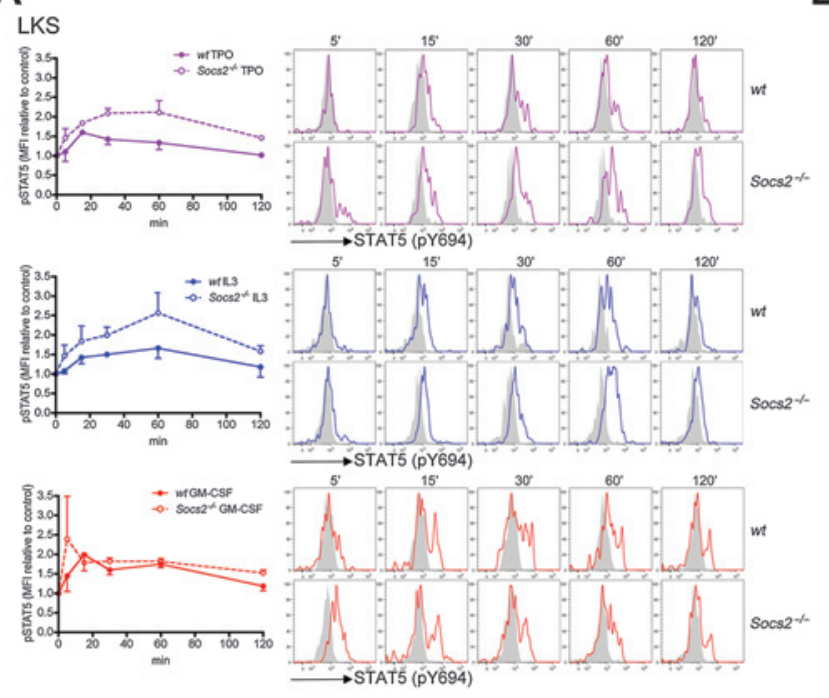

C

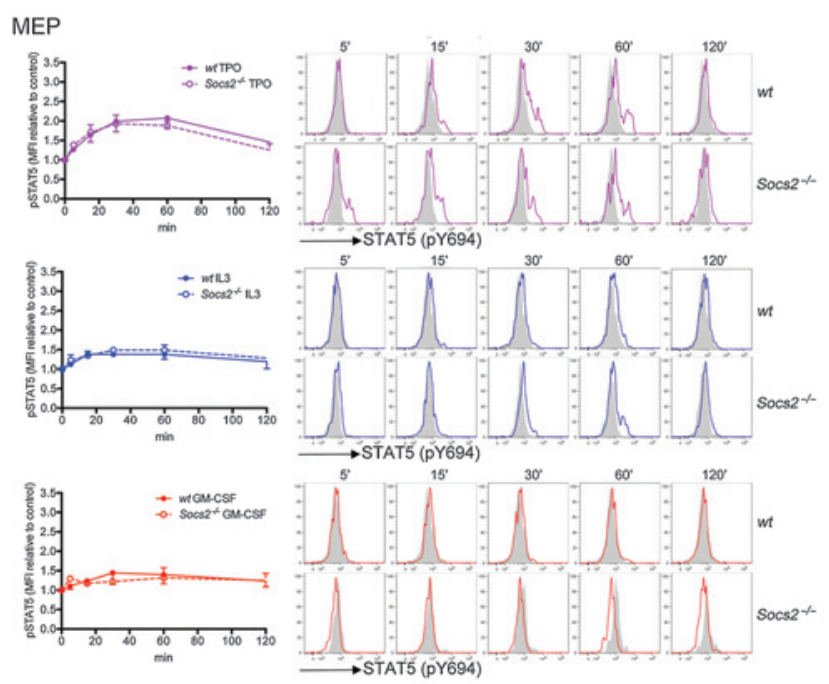

B

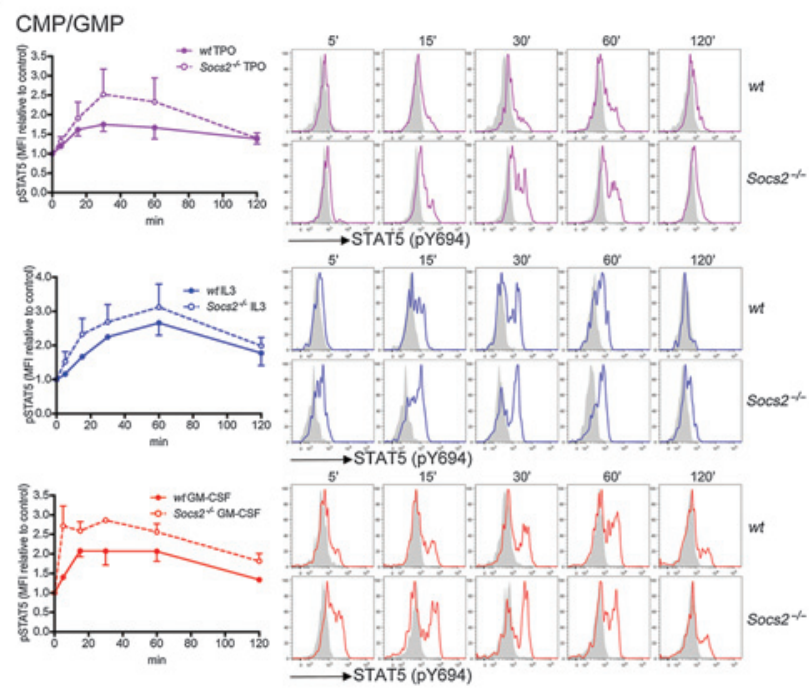

D
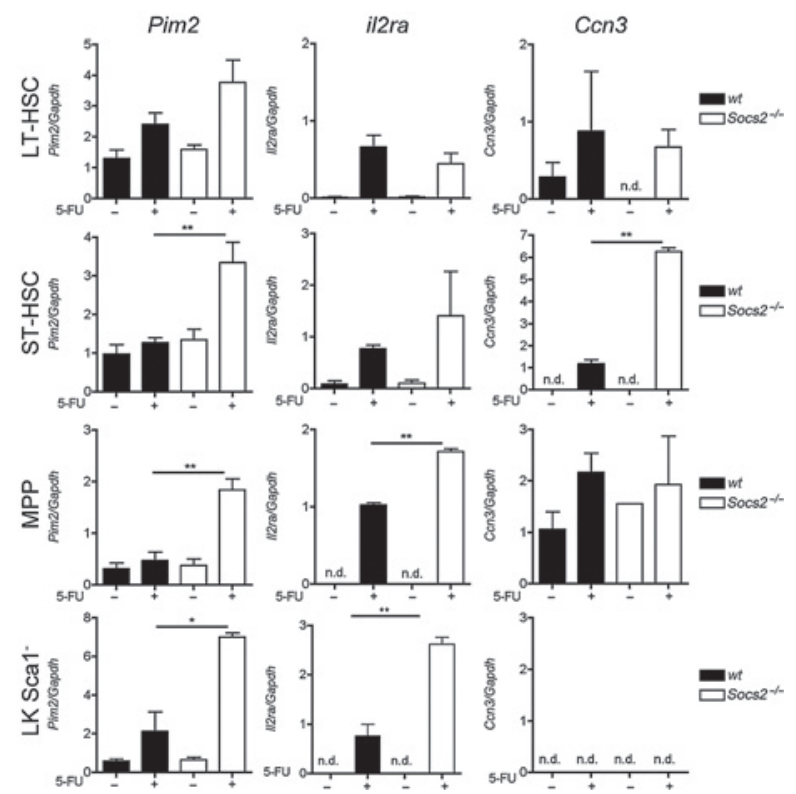

Figure 3.

SOCS2 controls STAT5 signaling in vitro and in vivo. A-C, total bone marrow cells isolated from wt and Socs ${ }^{-1-}$ mice were starved in complete medium with no serum for 2 hours prior to stimulation with the indicated cytokines for the indicated times. Nonstimulated samples were collected for each time point. Cells were then fixed immediately, and stained for surface antigens and for phospho-STAT5. Phospho-STAT5 expression in gated LKS (A), CMP/GMP cells $\left(\right.$ lin $^{-} \mathrm{c}-\mathrm{kit}^{+} \mathrm{Sca}-1^{-} \mathrm{CD} 34^{+} ; \mathrm{B}$ ), and MEP (lin ${ }^{-} \mathrm{c}-\mathrm{kit}^{+} \mathrm{Sca}--^{-} \mathrm{CD} 34^{-} ; \mathrm{C}$ ) was evaluated. Mean fluorescence intensity (MFI) relative to unstimulated control samples are plotted in the graphs on the left and representative histograms are shown on the right. The stimulation with each cytokine was repeated in three independent experiments, each with three mice/group. D, LT-HSC (lin ${ }^{-}$c-kit ${ }^{+}$Sca- $1^{+}$CD34 $4^{-}$Flt3 ${ }^{-}$), ST-HSC $\left(\right.$lin $^{-}$c-kit ${ }^{+}$Sca- ${ }^{+}$CD34 ${ }^{+}$Flt3 ${ }^{-}$), MPP (lin ${ }^{-}$c-kit ${ }^{+}$Sca- $1^{+}$CD34 ${ }^{+}$Flt3 ${ }^{+}$), and in $^{-} \mathrm{c}-\mathrm{kit}^{+} \mathrm{Sca}-1^{-}$were sorted from bone marrow of $w t$ and Socs $2^{-1-}$ mice 6 days after 5-FU treatment and qPCR for the indicated probes were performed. Results are from three independent sorting replicates, each starting from a pool of 6 mice for untreated and 10 mice for 5-FU-treated samples and demonstrate that SOCS2 is able to limit the transcription of STAT5 target genes induced upon 5-FU treatment.

presence of IL3, IL6, SCF, Flt3L, and TPO cytokine cocktail, or of each single cytokine, and measured the LKS expansion. Socs $2^{-/-}$LKS proliferative response to the cocktail, as well as to IL3 alone, exceeded that of $w t$ LKS (Fig. 2C). Moreover, when cultivated in methylcellulose-based medium containing SCF, IL3, IL6, and EPO, Socs $2^{-1-}$ bone marrow cells produced higher numbers of colony-forming units (CFU) than the wt counterpart (Fig. 2D). Altogether, these results demonstrate that
SOCS2 induction by cytokine stimulation limits HSC proliferative response.

\section{SOCS2 controls STAT5 signaling in vitro and in vivo}

Considering that STAT5 signaling is activated by TPO, IL3, and GM-CSF and promotes HSC self-renewal (26) and that, according to motif gene sets from MSigDB collection, SOCS2 promoter $(-2$ $\mathrm{kb},+2 \mathrm{~kb}$ around transcription start site) contains putative STAT5A 


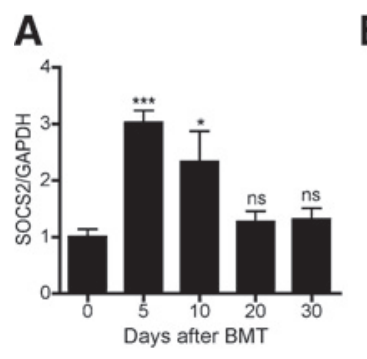

D

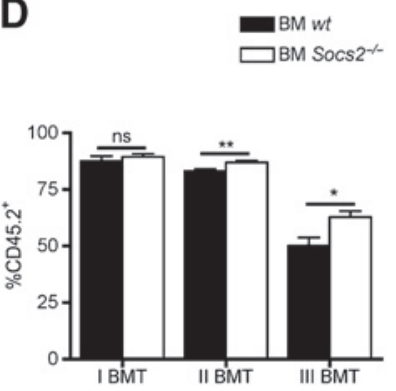

G

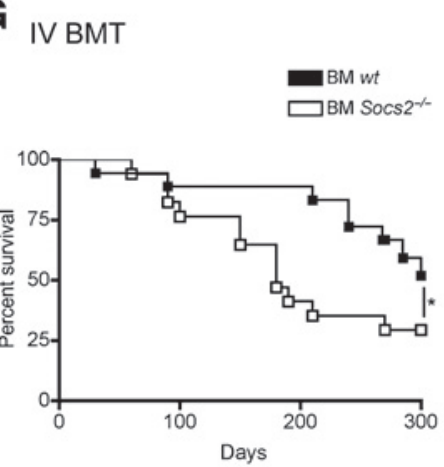

B вM wt

E
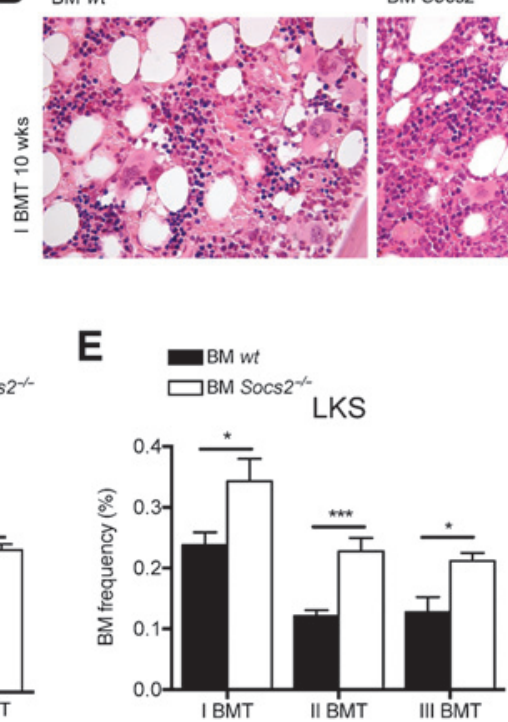

H

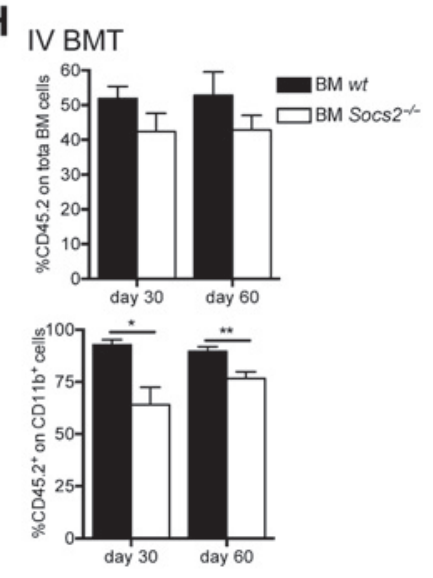

BM Socs $2^{--}$

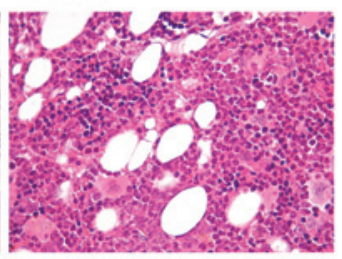

C

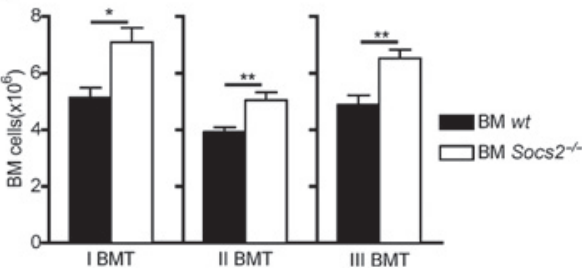

F
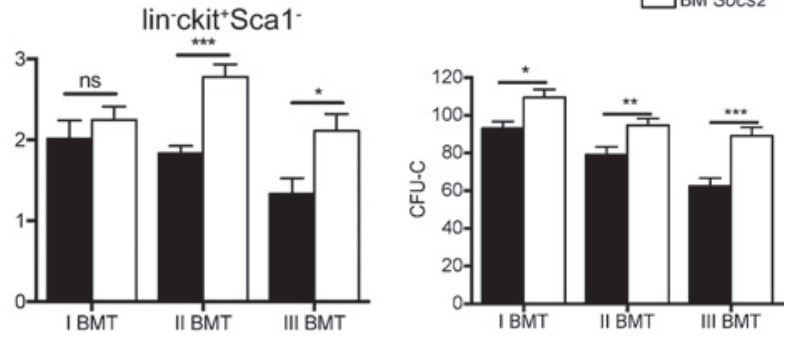

I IV BMT, day 300

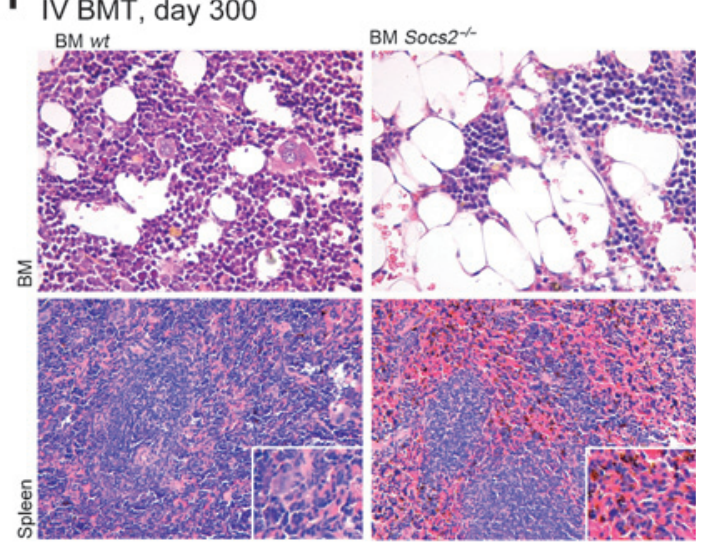

J

III BMT, 10 wks
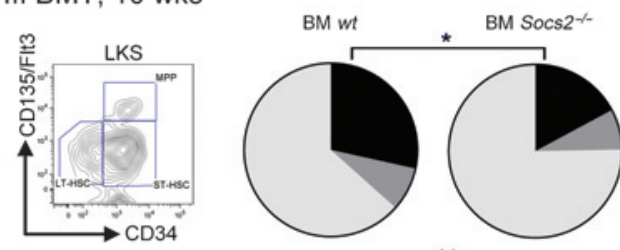

믄.HSC(*) 吕 ${ }_{M P P(*)}^{\text {ST.HSC }}$
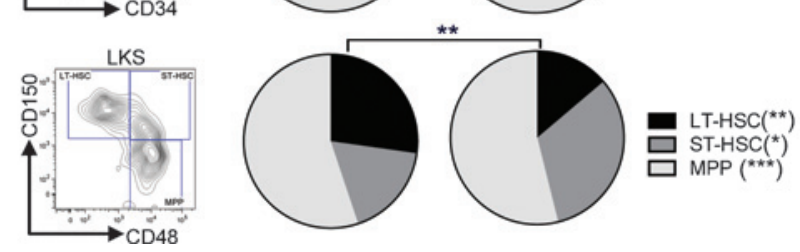

K

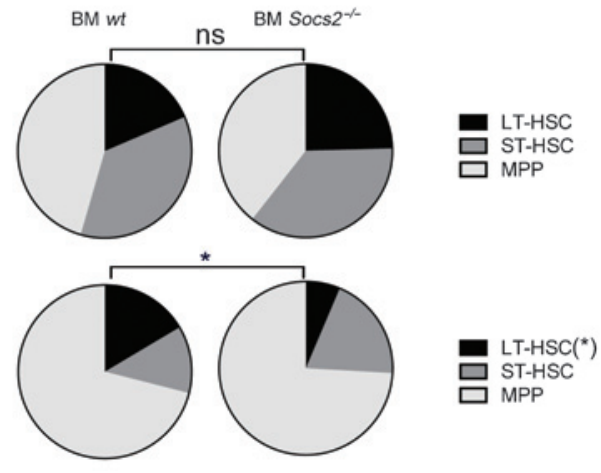

Figure 4.

Transient enhancement and long-term exhaustion of HSC function in serial BMT in the absence of SOCS2. A, Socs2 was transiently upregulated in Lin ${ }^{-}$bone marrow cells following BMT as revealed by qPCR (5 mice/group). B, histologic examination of bone marrow sections 10 weeks after I BMT showed enhanced repopulation of bone marrow cells in Socs $2^{-1-}$ compared with wt bone marrow recipients. C, bone marrow cells from one femur were counted by two operators 10 weeks after each BMT and averaged. D, engraftment of I, II, and III BMT recipients of Socs $2^{-/-}$bone marrow was enhanced, as evaluated by FACS as percentage of CD45.2 $2^{+}$cells in blood samples. E, higher frequency of LKS and lin ${ }^{-} \mathrm{C}^{-K i t^{+}}{ }^{+} \mathrm{Sca}-1^{-}$precursors in mice receiving Socs ${ }^{-/-}$bone marrow. F, bone marrow recovered from recipients receiving Socs $2^{-/-}$bone marrow formed higher numbers of CFU-C; G, reduced survival of IV BMT mice receiving Socs ${ }^{-/-}$bone marrow; results are pooled from three independent experiments with a total number of 17 mice/group; statistical significance was calculated with the log-rank test. $\mathrm{H}$, loss of reconstitution ability in the absence of SOCS2 in IV BMT recipients at the indicated times. (Continued on the following page.) 
but not STAT1, STAT3, STAT4, STAT5B, and STAT6 motifs (Supplementary Table S1), we hypothesized that SOCS2 could function as a negative feedback for STAT5 signaling in HSC upon TPO, IL3, or GM-CSF stimulation. In $w t$ LKS, TPO, IL3, and GM-CSF induced rapid phosphorylation of STAT5, peaking 15 minutes after stimulation and rapidly declining to baseline within 120 minutes (Fig. 3A, solid line; refs. 27, 28). Differently, in Socs $2^{-1-}$ LKS, STAT5 phosphorylation was enhanced and persisted beyond 120 minutes following stimulation with TPO or IL3 (Fig. 3A, dotted line), while a slight anticipation of STAT5 phosphorylation occurred upon GMCSF stimulation. Enhanced STAT5 phosphorylation was also evident in Socs $2^{-1-}$ CMP/GMP in response to TPO and GM-CSF, whereas only slight differences were induced by IL3 (Fig. 3B). In MEP, only TPO induced phospho-STAT5 but no differences were found between $w t$ and Socs $2^{-1-}$ genotypes (Fig. 3C).

We subsequently tested whether deregulated STAT5 signaling occurred in vivo in response to 5-FU, accounting for the increased myelopoietic response. LT-HSC, ST-HSC, MPP, and LK Sca- ${ }^{-}$ were sorted from $w t$ and Socs $2^{-/-}$bone marrow 6 days after 5-FU administration and the expression of the STAT5 target genes (23) Pim2, Il2ra, and Ccn3/Nov was evaluated (17). In the absence of SOCS2, the expression of Pim2 and Il2ra was higher in ST-HSC, MPP, and $\mathrm{Lin}^{-} \mathrm{CKit}^{+} \mathrm{Sca}-1^{-}$and that of Ccn3/Nov in ST-HSC (Fig. 3D). Interestingly, no differences were observed in LT-HSC, again suggesting that SOCS2 influences differentiating precursors rather than primitive HSC.

Altogether, these results indicate that in HSC, SOCS2 acts as a negative regulator of STAT5 signaling.

SOCS2 deficiency enhances transient HSC repopulating activity and causes exhaustion of long-term hematopoietic stemness

Considering that HSC proliferation and stemness maintenance are inversely correlated programs, we analyzed the impact of SOCS2 deficiency in the setting of repeated bone marrow hematopoietic stress adopting serial BMT. These experiments would let us evaluate whether the augmented expansion of ST-HSC and MPP and not of LT-HSC observed in Socs $2^{-1-}$ mice under 5-FU pressure could result in the impairment of LT-HSC functions. According to the upregulation of Socs 2 in hematopoietic precursor in settings of bone marrow stress, Socs 2 was transiently upregulated in Lin $^{-}$ bone marrow cells following BMT (Fig. 4A).

To perform serial BMT, two cohorts of lethally irradiated (CD45.1) mice received $1 \times 10^{6}$ total bone marrow cells from $w t$ or Socs $2^{-1-}$ littermates (CD45.2). Ten weeks after primary BMT (I $\mathrm{BMT}$ ), bone marrow cells from the chimeric mice were retransplanted into secondary (II BMT) lethally irradiated hosts (CD45.1). The same procedure was adopted for tertiary and quaternary transplants (III and IV BMT).

Consistent with enhanced stress response of Socs $2^{-/-}$HSC, the repopulation of bone marrow hematopoietic parenchyma evaluated histologically after the I BMT was significantly enhanced in Socs $2^{-/-}$bone marrow recipients than $w t$ recipients (Fig. 4B), as well as the degree of myelopoiesis within the splenic red pulp (Supplementary Fig. S2). Bone marrow cellularity (Fig. 4C) and bone marrow engraftment (Fig. 4D), assessed as percentage of circulating donor-derived CD45.2 $2^{+}$cells 10 weeks after BMT, were higher in mice receiving Socs $2^{-/-}$bone marrow compared with mice receiving $w t$ bone marrow in I, II, and III BMT. These effects were associated to higher frequencies of $\mathrm{LKS}$ and $\mathrm{Lin}^{-} \mathrm{CKit}^{+} \mathrm{Sca}-1^{-}$ precursors (Fig. 4E) and with enhanced in vitro colony-forming capacity in recipients of Socs $2^{-1-}$ bone marrow than of $w t$ counterpart (Fig. 4F). Within the $\mathrm{Lin}^{-} \mathrm{CKit}^{+} \mathrm{Sca}-1^{-}$subset, the distribution of CMP, GMP, and MEP was conserved in the two groups of mice (Supplementary Fig. S3).

Enhanced hematopoietic responses observed in mice receiving Socs $2^{-1-}$ bone marrow in BMT from I to III, in turn, resulted in the exhaustion of LT-HSC repopulating potential in the IV BMT, which caused reduced survival of mice receiving Socs $2^{-/-}$bone marrow compared with those receiving $w t$ bone marrow (Fig. $4 \mathrm{G}$ ). Such failure in bone marrow hematopoiesis was associated with a lower fraction of circulating donor hematopoietic cells $\left(\mathrm{CD} 45.2^{+}\right.$), particularly of the $\mathrm{CD} 11 \mathrm{~b}^{+}$compartment (Fig. $4 \mathrm{H}$ ). Further confirming the loss of repopulating potential of Socs $2^{-1-}$ bone marrow at the IV BMT, bone marrow histopathology on long-term survivals (300 days after IV BMT) revealed hypocellular marrow with scant foci of residual hematopoiesis in mice receiving Socs $2^{-/-}$bone marrow in comparison with mice receiving $w t$ bone marrow, which showed a more conspicuous bone marrow repopulation and diffuse hematopoietic cellularity (Fig. 4I, top). Consistently, the spleen red pulp of IV BMT with Socs $2^{-1-}$ donor bone marrow showed hemorrhagic appearance with foci of hemosiderin deposition underlining the failure of the splenic hematopoiesis, which was preserved in recipients of $w t$ bone marrow (Fig. 4I, bottom).

The experiments with 5-FU indicated that in the absence of SOCS2 LKS, specifically the committed progenitors ST-HSC and MPP were expanded with consequent reduction in relative frequency of LT-HSC (see Fig. 1). Also in III BMT recipients receiving Socs $2^{-1-}$ bone marrow, the increased frequency of LKS associated with expanded MPP and/or ST-HSC populations and with reduced frequency of LT-HSC, and this result was valid using both the SLAM markers and CD34/FLT3 to identify the populations (Fig. 4J). Such contraction of the LT-HSC compartment in mice receiving Socs $2^{-1-}$ bone marrow was progressively acquired along with the series of transplantations, with significant differences in LT-HSC frequencies starting from the II BMT (Fig. 4K). Therefore, the absence of SOCS2 caused initial enhanced bone marrow reconstitution by deregulated expansion of ST-HSC/ MPP, with exhaustion of long-term hematopoietic stemness resulting in decreased survival of IV BMT recipients of Socs $2^{-1-}$ bone marrow.

\section{SOCS2 upregulation in human hematopoietic malignancies} identifies unfavorable acute leukemia subsets

Hematopoietic stemness and differentiation programs are profoundly altered in hematopoietic malignancies, which develop from genetic aberrancies in a setting of a disrupted hematopoietic homeostasis. Following the demonstration that SOCS2 plays a

(Continued.) Results are representative of three experiments ( $n=6 /$ group). I, histologic examination of long-time survivors of IV BMT showing severe bone marrow loss and splenic populations in Socs $2^{-/-}$compared with wt bone marrow recipients. J, representative plot and relative frequency of LT-HSC, ST-HSC, and MPP calculated using CD34/FIt3 (top) or CD150/CD48 (bottom) in III BMT recipients of Socs2 ${ }^{-1-}$ bone marrow 10 weeks after BMT. K, the same analysis using SLAM markers is shown for I BMT and II BMT recipients 10 weeks after transplantation. All results are expressed as mean and SEM and are representative of four independent experiments ( $n=7 /$ group). 
role in regulating hematopoietic stress responses and in preserving HSC stemness maintenance in vivo, we challenged the hypothesis that its expression could be deregulated in human hematopoietic malignancies. Analyzing public GEP, we found significant differences in SOCS2 expression in human hematopoietic neoplasms with different degree of differentiation, namely CML, myelodysplastic syndromes (MDS), AML, and ALL (ANOVA, $P<0.001$ ), with SOCS2 levels peaking in some AML and in most ALL cases (Fig. 5A). These results were confirmed by in situ quantitative immunolocalization analysis of SOCS2 (Fig. 5B). Specifically, in normal bone marrow, SOCS2 stained progenitors populating para-trabecular areas and myeloid precursors in the interstitium of the inter-trabecular areas (Fig. 5C, black arrows). No or faint expression was detected in mature cells (blue arrows). In CML, SOCS2 expression was increased in the expanded myeloid precursors (black arrows) and decreased in clusters of differentiated myeloid elements (blue arrows). In MDS, characterized by ineffective hematopoiesis of the neoplastic clone, SOCS2 marked immature precursors also showing abnormal localization (black arrows), whereas in AML and ALL, it was expressed in the blastic population effacing the bone marrow. Thus, the levels of both SOCS2 transcript and protein were associated with the immature precursor populations expanding at the expense of the effective hematopoietic pool.

Considering SOCS2 heterogeneity, we analyzed how SOCS2 expression was modulated in each leukemic subtype. In $\mathrm{CML}$, SOCS2 mRNA and protein (Supplementary Fig. S4) increased along progression from chronic phase toward accelerated phase and blast crisis.

In AML patients, high levels of SOCS2 characterized clones with specific cytogenetic lesions such as those with MLL (11q23) and BCR/ABL1 (t9:22) lesions, which share a dismal prognosis (Fig. 5D). Consistently, elevated SOCS2 levels correlated with reduced overall survival (OS) in AML patients (Fig. 5E), also within subsets of AML with normal karyotype enriched in clones with FLT3-ITD and associated with poor prognosis (Fig. 5F).

In ALL, high SOCS2 characterized more immature subsets, namely c-ALL/pre-B-ALL or pro-B-ALL, whereas levels of SOCS2 closer to that of normal bone marrow were detected in more mature B-ALL and in T-ALL (Fig. 5G). Grippingly, also in ALL, the same subsets with unfavorable cytogenetics, namely those with MLL abnormalities and those characterized by BCR/ABL1, displayed the highest levels of SOCS2 (Fig. 5H).

SOCS2 marks a stemness-related program in acute leukemias and is part of IKZF1/Ikaros and MEF2C regulatory networks

AML-associated HSC and leukemic stem cell (LSC) signatures have been recently described and related to bad prognosis (29). Considering that the basal expression of SOCS2 is confined to primitive HSC and that the upregulated SOCS2 expression characterized unfavorable acute leukemias, we hypothesized that SOCS2 could be part of a stemness program in acute leukemias. To this aim, we generated a list of genes positively correlated with SOCS2 in acute leukemias (Supplementary Table S2) and crossmatched the list with the AML HSC/LSC signature (29). We found a high degree of overlap between the two gene lists $(P<0.001$; Fig. $6 \mathrm{~A})$ and the shared genes, namely TRAF3IP2, MEF2C, FRMD4B, BCL11A, COL5A1, ELK3, ERG, PTK2, SPTBN1, and ARPP-19, corresponding to 32 probe sets, were used to generate a more restricted gene list of "leukemic stemness- and SOCS2-associated gene list" (Table 1).
Unsupervised clustering of acute leukemia samples according to this restricted gene list was suitable to group acute leukemias with SOCS2 levels above (or below) the median value (SOCS2 $2^{50-100}$ ), comprising most ALL and a small subgroup of AML (Fig. 6B). Specifically within AML samples, cases characterized by MLL (11q23) and $\mathrm{t}(15 ; 17)$ cytogenetic lesions formed defined clusters associated with high or low SOCS2, respectively (Fig. 6C). Similarly, within ALL samples, the "leukemic stemness- and SOCS2associated gene list" pinpointed patients with E2A/PBX1 translocation and low SOCS2 expression and patients with MLL or BCR/ ABL aberrations and high SOCS2 expression (Fig. 6D).

We also interrogated the list of SOCS2-related genes to identify common regulatory networks shared by SOCS2 and its correlates. Following the hypothesis that deregulated SOCS2 expression could depend on STAT5 activation, we searched for known STAT5 targets identified in HSC $(17,30)$. More specifically, only two of the 99 SOCS2 correlates belonged to STAT5-predicted targets (Fig. $6 \mathrm{E}$ ) according to $\mathrm{C} 3$ motif genesets collection of MSigDB (Supplementary Table S1), suggesting that upregulated SOCS2 was not a mere bystander result of JAK2-STAT5 activation.

Rather, we noted that several SOCS2 best correlates, including CTGF, MEF2C, DNTT, CD79B, ADD3, CD24, LIG, BLNK, and SOCS2 itself, were predicted or validated target genes of the transcriptional factor IKZF1/Ikaros, which plays key roles in normal hematopoiesis and B-cell lymphopoiesis (31) and that is involved in AML (32), ALL (33) and CML blast crisis (34). Moreover, $M E F 2 C$, encoding the transcription factor myocyte enhancer factor 2C (MEF2C), is one of the best correlates of SOCS2 and is also a target of Ikaros. MEF2C has similar expression pattern of SOCS2 in normal HSC (35) and is ectopically expressed in MLL-rearranged AML (36). Therefore, we evaluated whether a regulatory network existed involving SOCS2, Ikaros and/or $\mathrm{MEF} 2 \mathrm{C}$ in acute leukemias by ARACNe reverse engineering analysis (37). We determined transcriptional networks of IKZF1/ Ikaros and MEF2C in bone marrow myeloid and lymphoid precursors, AML and ALL, and identified genes whose expression was significantly related with them; these genes were defined as "first neighbors" of the network representing putative transcriptional targets of IKZF1/Ikaros and MEF2C (Supplementary Tables S3 and S4). Of note, SOCS2 was one of the first neighbor of both IKZF1/Ikaros and MEF2C (Supplementary Tables S3 and S4) in normal HSC and acute leukemias, but not in mature B cells, and related malignancies (non-Hodgkin lymphomas), confirming the specificity of these molecular interactions in hematopoietic precursor malignancies (Supplementary Tables S5 and S6). MEF2C was a target of IKZF1/Ikaros sharing with IKZF1 a network of 530 targets (Supplementary Fig. S5). Among the SOCS2 correlates that were identified as first neighbors, 84 of 87 IKZF1/Ikaros putative targets were also MEF2C targets, whereas other 90 genes were exclusively MEF2C targets (Fig. 6F and G), suggesting a more direct dependency of SOCS2 on MEF2C.

Common SOCS2-correlated programs are engaged in human acute leukemias and in in vivo experimental HSC stress

Prompted by in situ analyses of human acute leukemias revealing the link between SOCS2 and, primarily, MEF2C, we investigated whether such link exists also in a condition of hematopoietic stress in vivo. To this aim, we generated GEP of murine LKS sorted from $w t$ and Socs $2^{-1-}$ bone marrow 6 days after 5-FU treatment. Supervised comparison revealed 51 differently expressed genes (DEG) with a fold change $\geq 2$ and $P<0.001$ 
Figure 5.

High expression of SOCS2

characterizes unfavorable acute leukemia subsets. A, analysis of

SOCS2 using public GEP datasets of normal bone marrow, MDS, CML, AML, and ALL samples revealed

upregulated SOCS2 levels in leukemias compared with normal bone marrow. Box plot with quartiles, minimum, and maximum values are shown; the Benjamini-Hochberg correction was applied and statistical analysis was performed using a oneway ANOVA. B, ImageJ quantification of in situ expression of SOCS2 in bone marrow samples from normal bone marrow, MDS, CML, AML, and ALL; statistical analysis was performed using a one-way ANOVA; each pathologic condition was also compared with normal bone marrow with an unpaired $t$ test. C,

representative images of in situ expression of SOCS2 in bone marrow samples from normal bone marrow, MDS, CML, AML, and ALL; scale bars, $50 \mu \mathrm{m}$. D, higher SOCS2 levels corresponded to the unfavorable AML subset carrying t(11q23)/MLL and $\mathrm{t}(9 ; 22)$ karyotypes; boxes and statistics are calculated as above. E, the Kaplan-Meier analysis of OS of AML patients according to SOCS2 expression, grouped in quartiles. High soCS2 expression correlated with poorer OS; statistical analysis was performed using the log-rank test. F the same analysis as in E was applied to AML cases with normal karyotype. $G$, higher expression of SOCS2 in ALL identified more immature subgroups (pro-B- or pre-B-ALL) rather than mature B- and T-ALL. H, expression of SOCS2 in ALL cases according to the karyotype; box plot and statistics are calculated as above.
A

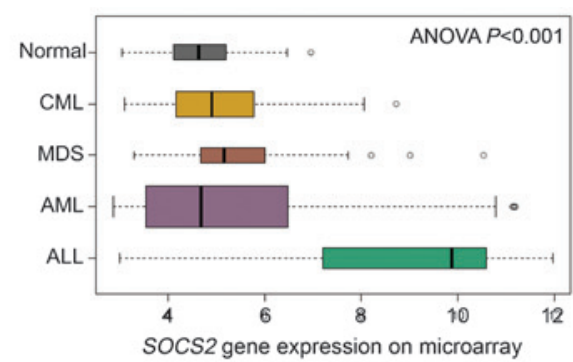

B

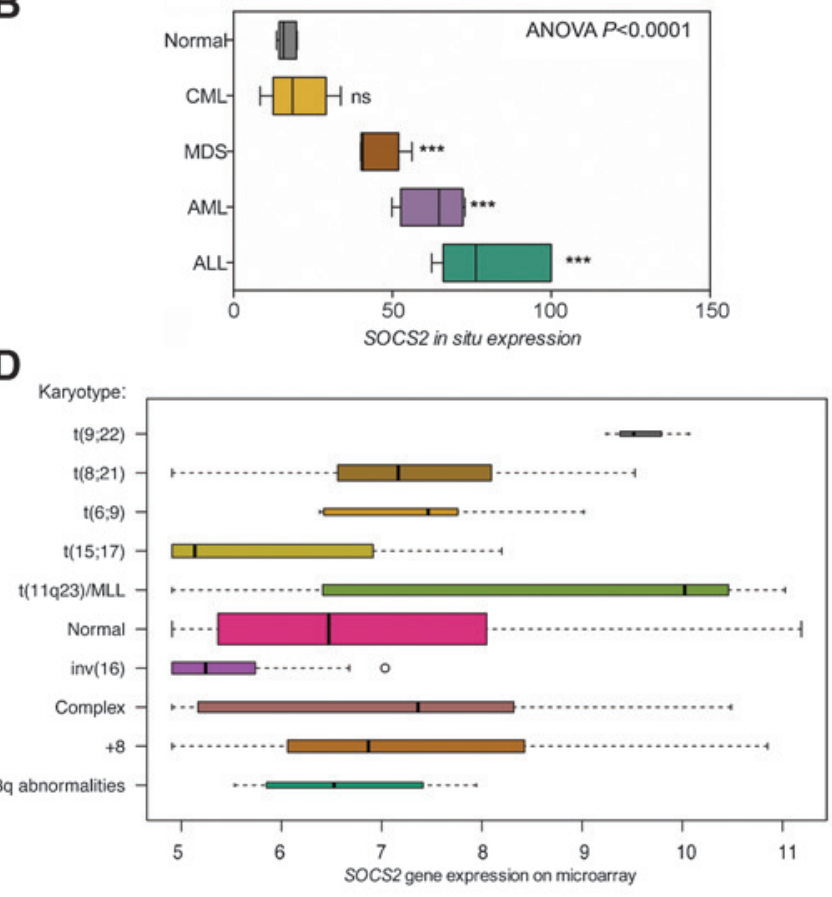

E

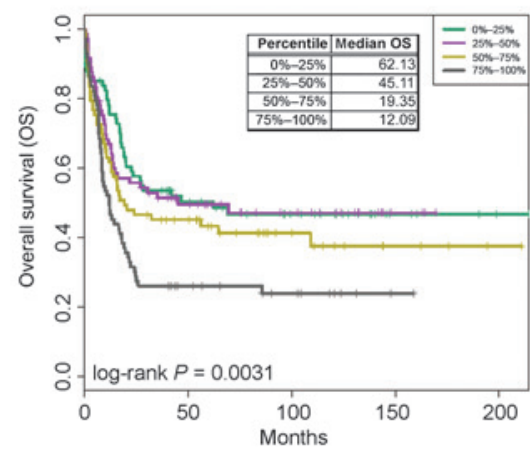

G

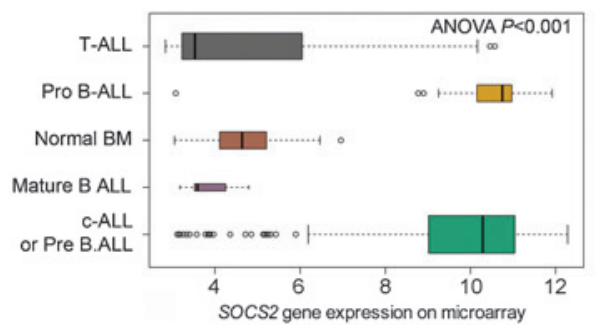

C

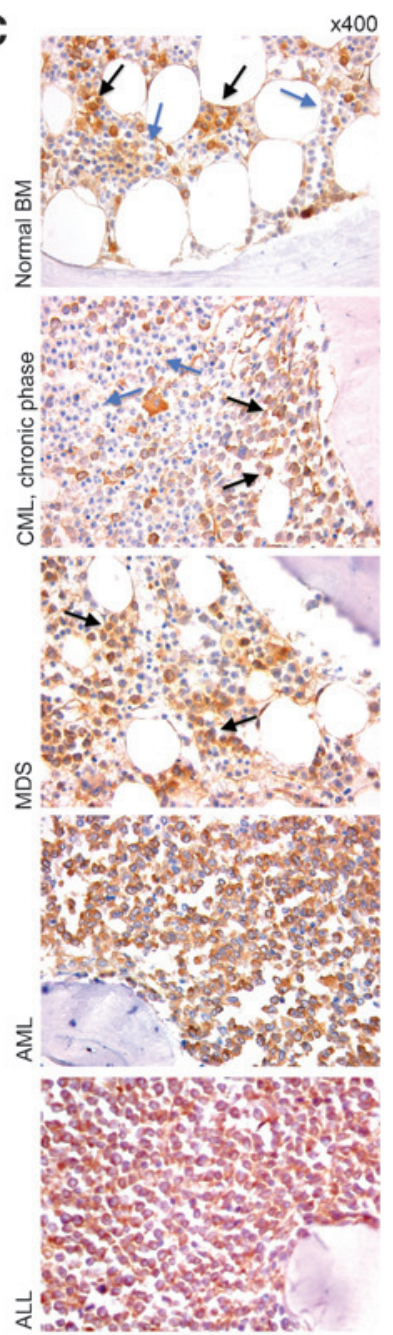

$\mathbf{F}$

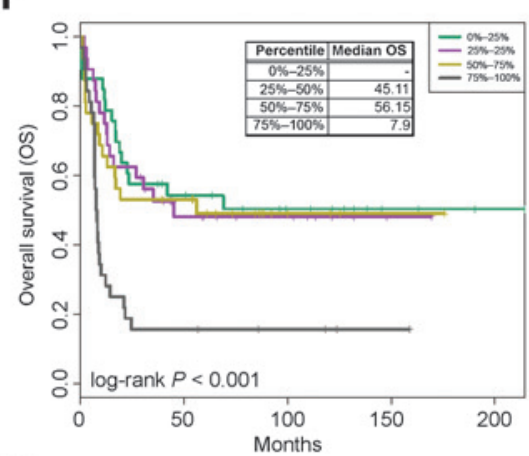

H

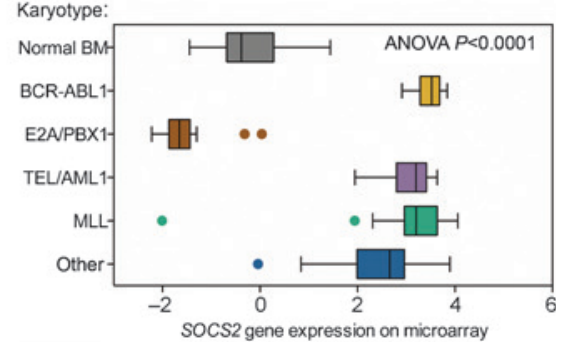


A

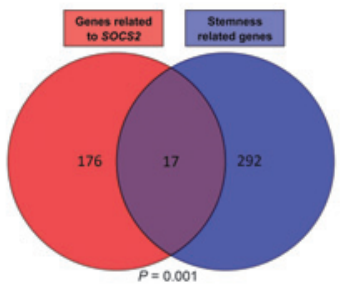

B
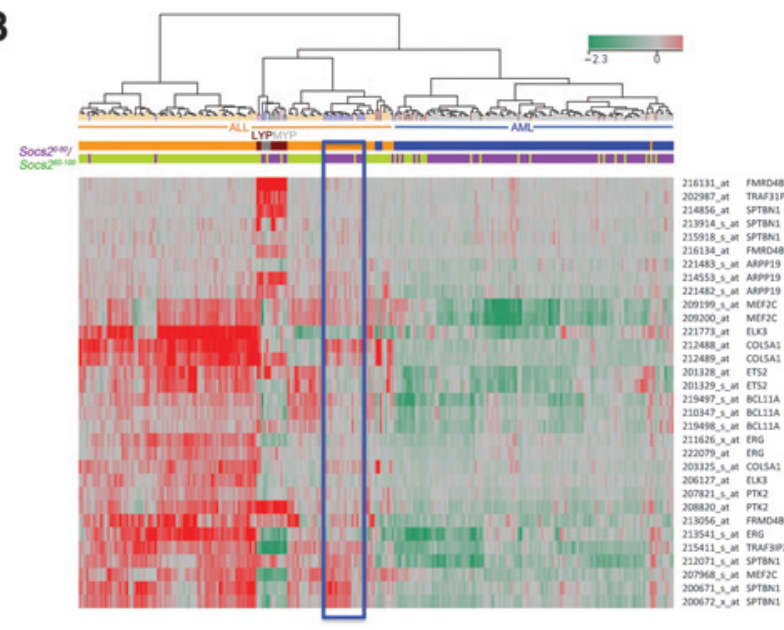

C

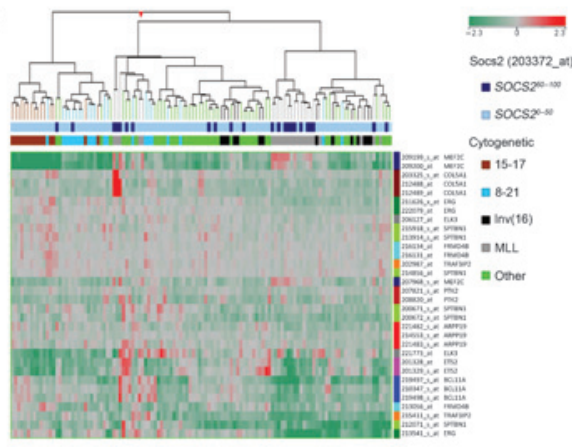

E

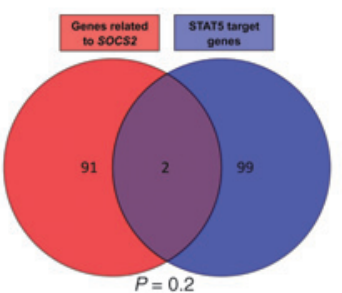

G

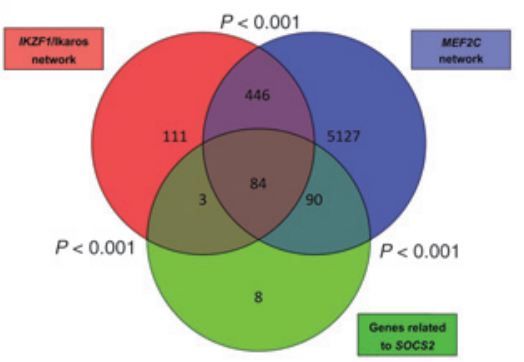

H

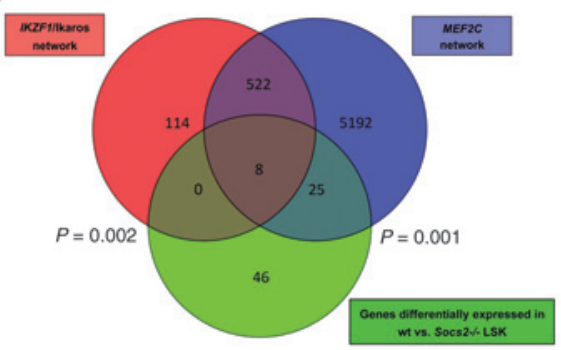

D

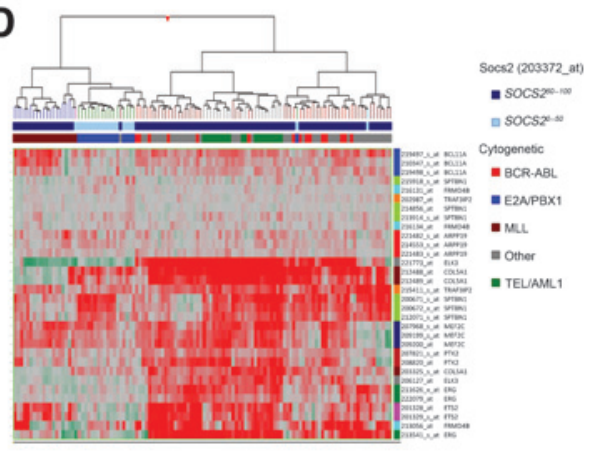

$\mathbf{F}$

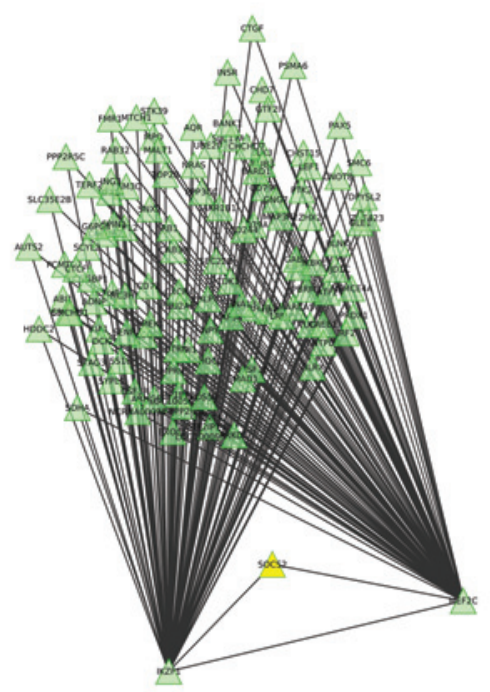

I
Figure 6.

In acute leukemias, SOCS2 and its correlated stem cell signature cluster specific subtypes and are part of regulatory networks independent from STAT and dependent on Ikaros and MEF2C. A, Venn diagram showing significant overlap between SOCS2 correlated genes and AML stemnessrelated genes. $B$, unsupervised hierarchical clustering of $A M L$ and $A L L$ samples according to the gene list indicated in Table 1 showed clear enrichment of ALL cases and of a subset of AML cases in the group with higher SOCS2 expression (Socs $^{50-100}$ ). LYP, lymphoid precursor, MYP, myeloid precursors. $C$, the same analysis, applied to AML samples subdivided according to cytogenetic lesions, was able to identify MLL- and t(15;17)-rearranged samples expressing high and low SOCS2, respectively. D, the same analysis as in panel B for ALL samples clustered subsets with MLL and E2A/ PBX1 lesions within high and low SOCS2 groups, respectively. E, Venn diagram showing the lack of STAT5 potential targets within the SOCS2 correlated gene list; the Fisher exact test was used for statistical analysis. F, regulatory network of two transcription factors Ikaros and MEF2C as defined by ARACNe algorithm on top 100 genes sorted by mutual information score. Each gene is represented by a green triangle and each interaction is represented by a black line. G, Venn diagram showing significant overlaps between IKZFI/ Ikaros network, MEF2C network, and SOCS2-related genes; the Fisher exact test was used for statistical analysis. $\mathrm{H}$, Venn diagram showing the overlap of IKZFI/Ikaros first neighbors, MEF2C

first neighbors, and 51 genes differently expressed in Socs2 $2^{-1}$ versus $w t$ murine LKS 6 days after 5FU treatment; the Fisher exact test was used for statistical analysis. I, murine bone marrow Lin ${ }^{-}$cells were infected with the lentiviral vectors containing Mef $2 c$ and $/ k z f 1$ ORF or with empty vector as negative control and qPCR was performed for Socs2; noninfected cells were included in the analysis as negative control and Lin cells grown in the presence of the cytokine cocktail were used as positive control of Socs 2 induction. 
Table 1. Gene list of SOCS2 best correlates associated to stemness

\begin{tabular}{|c|c|}
\hline Probe set ID & Gene symbol \\
\hline 214553_s_at & ARPP19 \\
\hline 221482_s_at & ARPP19 \\
\hline 221483_s_at & ARPP19 \\
\hline 210347_s_at & $B C L 11 A$ \\
\hline 219497_s_at & $B C L 11 A$ \\
\hline 219498_s_at & $B C L 11 A$ \\
\hline 203325_s_at & COL5A1 \\
\hline 212488_at & COL5A1 \\
\hline 212489_at & COL5A1 \\
\hline 206127_at & ELK3 \\
\hline 221773_at & $E L K 3$ \\
\hline 211626_x_at & $E R G$ \\
\hline 213541_s_at & $E R G$ \\
\hline 222079_at & $E R G$ \\
\hline 201328_at & ETS2 \\
\hline 201329_s_at & ETS2 \\
\hline 213056_at & $F R M D 4 B$ \\
\hline 216131_at & $F R M D 4 B$ \\
\hline 216134_at & $F R M D 4 B$ \\
\hline 207968_s_at & MEF2C \\
\hline 209199_s_at & MEF2C \\
\hline 209200_at & MEF2C \\
\hline 207821_s_at & PTK2 \\
\hline 208820_at & PTK2 \\
\hline 200671_s_at & SPTBN1 \\
\hline 200672_x_at & SPTBN1 \\
\hline 212071_s_at & SPTBN1 \\
\hline 213914_s_at & SPTBN1 \\
\hline 214856_at & SPTBN1 \\
\hline 215918_s_at & SPTBN1 \\
\hline 202987_at & TRAF3IP2 \\
\hline 215411_s_at & TRAF3IP2 \\
\hline
\end{tabular}

(Supplementary Table S7). Among them, eight were upregulated and 43 were downregulated in Socs $2^{-1-}$ as compared with $w t$ counterpart.

In the absence of SOCS2, there was a positive enrichment of gene sets encoding for proteins involved in the translational machinery, in line with a more active metabolism of Socs $2^{-1-}$ LKS in response to myeloablation, whereas among the pathways negatively enriched in Socs $2^{-1-}$ LKS, many had relevance in cancer and others were linked to cytokine response (Supplementary Table S8 and Supplementary Fig. S6).

We then investigated similarities between gene programs differentially regulated in human leukemias according to SOCS2 gene expression and the 51 DEG of murine LKS under hematopoietic stress. We documented a significant correspondence between the cellular programs enriched in the two signatures as defined by GSEA. In particular, 10 "curated genesets" (Supplementary Table S9) and 7 "oncogenic signatures" (Supplementary Table S10) were enriched using the 51 DEG and 2 out of 10 of the former and seven out of eight of the latter categories were also enriched considering SOCS2-correlated genes in human leukemias (Supplementary Tables S9 and S10, yellow highlights).

Moreover, considering the 79 probe sets corresponding to the 36 out of 51 murine DEG that are detectable in the human arrays, 33 were MEF2C putative targets identified by reverse engineering in human acute leukemias (corresponding to 11 genes), and only 8 of these 33 probe sets (corresponding to SOCS2 itself and PATZ1 genes) were also IKZF1/Ikaros first neighbors (Fig. 6H).

Collectively, human reverse engineering and murine GEP results were strongly indicative that MEF2C could be a key regulator of SOCS2 expression in HSC in different setting, while suggesting a less prominent or indirect role for Ikaros. To confirm this potential mechanism, we lentivirally transduced murine bone marrow $\mathrm{Lin}^{-}$with Mef2c and Ikaros ORF and confirmed the expression of the two transcripts by qPCR (Supplementary Fig. S7). The overexpression of MEF2C was able to significantly upregulate Socs 2 transcription, similarly to the induction mediated by cytokine cocktail stimulation, whereas Ikaros expression was unable to affect the transcription of Socs2. Therefore, in HSC, the expression of Socs2, besides being regulated by cytokine stimulation, is also controlled by MEF2C.

\section{Discussion}

HSC self-renewal and differentiation in response to hematopoietic stress are regulated by cytokines signaling through JAKSTAT pathways (38). Our report describes for the first time the active role of SOCS2 in regulating HSC functions during nonhomeostatic conditions such as bone marrow stress or malignant hematopoiesis. In this setting, we have identified a new regulatory mechanism inducing Socs 2 independently from STAT pathways while depending on MEF2C.

As negative regulator of the JAK2-STAT5 pathway, SOCS2 keeps in check stress-induced HSC response avoiding uncontrolled amplification of ST-HSC, MPP, and committed precursors thus preserving long-term hematopoiesis. Accordingly, we demonstrate that SOCS2 deficiency is associated with aberrant expansion of hematopoietic progenitors following 5-FU myeloablation and with the impoverishment of the stem cell pool in response to serial BMT. Indeed, SOCS2 deficiency enhanced bone marrow reconstitution in I, II, and III BMT recipients and, eventually, loss of long-term hematopoietic function, with decreased survival of IV BMT recipients. This was related with the composition of the LKS pool that in Socs $2^{-/-}$bone marrow chimeras was characterized by higher ST-HSC and MPP but reduced LT-HSC frequencies.

SOCS2 is overexpressed in primitive HSC (13-16) compared with more differentiated populations, both in human (13-16 and mouse $(22,25)$, suggesting that basal SOCS2 expression could contribute to stemness maintenance. Nevertheless, Hansen and colleagues (25) published that Socs2-deficient mice had normal reconstitution capacity in primary and secondary competitive BMT experiments. Our kinetic analysis of Socs 2 expression following the bone marrow myeloablation (BMT or 5-FU) clearly indicated that SOCS2 overexpression and SOCS2-negative regulation of STAT5 are transient and occur within 1 day after myeloablative treatment. Of note, the analysis of bone marrow composition reported by Hansen and colleagues (25) was performed 4 weeks after BMT, a time point at which bone marrow homeostatic hematopoiesis is restored.

We have dissected in vitro the dominant mechanism exerted by SOCS2 over HSC expansion: in conditions of hematopoietic stress or upon triggering with IL3, TPO, or GM-CSF, SOCS2 is transiently upregulated and primarily tunes JAK2-STAT5 activation, a pathway known to regulate cytokine-mediated HSC response (39) and self-renewal (26). Increased SOCS2 expression occurs also in bone marrow of patients treated with hematopoietic cytokines, suggesting a clear connection in hematopoietic cytokine response between mice and humans. However, we cannot exclude that in HSC other cytokines and/or other STATs could be regulated by SOCS2, nor that other SOCS family members, such as the known STAT5 regulators SOCS1 and SOCS3, could play 
redundant roles (6). Other pathways are also activated by hematopoietic cytokines such as the phosphatidylinositol-3-kinase (PI3K) pathway and the mitogen activated protein kinase (MAPK) pathway. This multiplicity of checkpoints is necessary to maintain bone marrow integrity and suggests that the differences observed in our experiments, although statistically significant, might be attenuated by redundancy of the system.

Altered Socs2 expression has emerged from GEP of several hematologic malignancies. In CML patients, upregulated SOCS2 was found in $\mathrm{CD} 34^{+}$cells during the transition from the chronic phase to the blast phase and has been associated with poor prognosis $(40,41)$. In contrast, a mouse model of BCR/ABL1induced CML shows the same incidence regardless SOCS2 deficiency (25). However, these data cannot be considered conclusive given that such model is characterized by a short latency and rapid progression, representing a suboptimal setting to appreciate any potential phenotype related with SOCS2 effects.

The significance of SOCS2 modulation in hematologic malignancies other than $\mathrm{CML}$, including MDS, AML, and ALL has never been thoroughly addressed. Our data confirm SOCS2 upregulation along different CML blast-enrichment phases, and document for the first time that SOCS2 increased expression characterizes specific AML and ALL subsets with unfavorable BCR/ABL or MLL lesions and with the mixed lineage phenotype, a feature of aberrant hematopoietic stemness. Of interest, ALL and SOCS2 have never been associated before. In AML, we could also demonstrate that high SOCS2 levels correlate with poor prognosis. At the molecular level, SOCS2 and the coregulated genes significantly overlapped with those belonging to AML-associated HSC and LSC signatures (29).

In patients carrying mutations and translocations associated with constitutive activation of JAK2/STAT5, such as BCR-ABL or FLT3-ITD, high SOCS2 possibly reflects the chronic phosphorylation of STAT5. Accordingly, the expression of SOCS2 is downregulated in CML patients with BCR-ABL following treatment with the tyrosine kinase inhibitor imatinib (42), and patients refractory to the treatment displayed higher SOCS2 compared with treatment-sensitive ones (41). Similarly, in AML patients carrying the FLT3-ITD mutation, induction of SOCS1, SOCS2, and SOCS3 is linked with STAT5 activation, in line with the concept of SOCS2 functioning as a negative regulator of FLT3 (43).

Our reverse engineering analysis for the first time suggests a novel SOCS2 regulatory network that is independent from STAT activation and that involves primarily MEF2C and less consistently Ikaros. At the steady state, MEF2C and SOCS2 expression patterns are overlapping, being high in LKS and CMP and downregulated in GMP and MEP; in hematologic diseases, MEF2C upregulation is described for AML with MLL lesions (those associated to highest SOCS2), where it contributes to confer stemness features (35). Ikaros is a transcriptional regulator that controls key events for lymphoid differentiation in MPP and its dominant-negative isoforms or deletions have been reported in cases of infant and childhood ALL $(44,45)$ as well as in blast crisis

\footnotetext{
References

1. Kondo M, Wagers AJ, Manz MG, Prohaska SS, Scherer DC, Beilhack GF, et al. Biology of hematopoietic stem cells and progenitors: implications for clinical application. Annu Rev Immunol 2003;21:759-806.

2. Passegue E, Wagers AJ, Giuriato S, Anderson WC, Weissman IL. Global analysis of proliferation and cell cycle gene expression in the regulation
}

of Philadelphia positive CML (34). Interestingly, GEP analysis of MEF2C competent versus deficient MPP indicated a direct relationship between MEF2C and Ikaros (46); however, such relationship has never been identified in acute leukemias neither it has been associated with SOCS2. Our GEP analyses clearly indicate that SOCS2-related transcriptional programs and MEF2C are functionally associated also in murine HSC under stress conditions. Moreover, we experimentally demonstrated that MEF2C expression in HSC is sufficient to upregulate Socs 2 even in the absence of cytokine stimulation.

Altogether, we have uncovered that SOCS2 is part of a transcriptional program with prognostic relevance in acute leukemias, and identified MEF2C as a novel key factor that is complementary to STAT5 in regulating SOCS2 expression in HSC. This novel MEF2C-mediated regulation of SOCS2 might exert a pivotal role in the absence of cytokine stimulation, such as in HSC at the steady state and the aberrant expression of MEF2C, SOCS2 and the correlated genes might confer leukemic stemness features to the neoplastic hematopoietic clones. Hence, this emerging network has clinical relevance and deserves further investigation.

\section{Disclosure of Potential Conflicts of Interest}

No potential conflicts of interest were disclosed.

\section{Authors' Contributions}

Conception and design: C. Vitali, M.P. Colombo, C. Tripodo Development of methodology: C. Vitali, S. Miotti

Acquisition of data (provided animals, acquired and managed patients, provided facilities, etc.): C. Bassani, C. Chiodoni, E. Fellini, C. Guarnotta, L. De Cecco, C. Tripodo

Analysis and interpretation of data (e.g., statistical analysis, biostatistics, computational analysis): C. Vitali, C. Chiodoni, E. Fellini, C. Guarnotta, S. Sangaletti, F. Fuligni, P.P. Piccaluga, C. Tripodo

Writing, review, and/or revision of the manuscript: C. Vitali, C. Guarnotta, P.P. Piccaluga, M.P. Colombo, C. Tripodo

Administrative, technical, or material support (i.e., reporting or organizing data, constructing databases): C. Bassani, C. Guarnotta

Study supervision: M.P. Colombo

\section{Acknowledgments}

The authors thank Andrea Vecchi for cell sorting and Matteo Dugo for GEP analysis. A special thanks to Ms. Margherita Sciarra Bottani for donating the LSR Fortessa in memory of her loved daughter Simonetta.

\section{Grant Support}

This work was supported by grants from Associazione Italiana per la Ricerca sul Cancro (AIRC Investigator Grant number 14194; M.P. Colombo); Program Innovative Tools for Cancer Risk Assessment and Diagnosis, $5 \times 1000$ grant number 12162; M.P. Colombo and C. Tripodo). C. Vitali is supported by Fondazione Umberto Veronesi young investigator fellowship.

The costs of publication of this article were defrayed in part by the payment of page charges. This article must therefore be hereby marked advertisement in accordance with 18 U.S.C. Section 1734 solely to indicate this fact.

Received December 10, 2014; revised February 25, 2015; accepted March 4, 2015; published OnlineFirst April 9, 2015.

of hematopoietic stem and progenitor cell fates. J Exp Med 2005;202 1599-611.

3. Harrison DE, Lerner CP. Most primitive hematopoietic stem cells are stimulated to cycle rapidly after treatment with 5-fluorouracil. Blood 1991; 78:1237-40. 
4. King KY, Goodell MA. Inflammatory modulation of HSCs: viewing the HSC as a foundation for the immune response. Nat Rev 2011;11:685-92.

5. Baker SJ, Rane SG, Reddy EP. Hematopoietic cytokine receptor signaling. Oncogene 2007;26:6724-37.

6. Alexander WS. Suppressors of cytokine signalling (SOCS) in the immune system. Nat Rev 2002;2:410-6.

7. Yoshimura A, Naka T, Kubo M. SOCS proteins, cytokine signalling and immune regulation. Nat Rev 2007;7:454-65.

8. Greenhalgh CJ, Rico-Bautista E, Lorentzon $\mathrm{M}$, Thaus $\mathrm{AL}$, Morgan PO, Willson TA, et al. SOCS2 negatively regulates growth hormone action in vitro and in vivo. J Clin Invest 2005;115:397-406.

9. Metcalf D, Greenhalgh CJ, Viney E, Willson TA, Starr R, Nicola NA, et al Gigantism in mice lacking suppressor of cytokine signalling-2. Nature 2000;405:1069-73.

10. Knosp CA, Carroll HP, Elliott J, Saunders SP, Nel HJ, Amu S, et al. SOCS2 regulates Thelper type 2 differentiation and the generation of type 2 allergic responses. J Exp Med 2011;208:1523-31.

11. Lee SH, Yun S, Piao ZH, Jeong M, Kim DO, Jung H, et al. Suppressor of cytokine signaling 2 regulates IL-15-primed human NK cell function via control of phosphorylated Pyk2. J Immunol 2010;185:917-28.

12. Posselt G, Schwarz H, Duschl A, Horejs-Hoeck J. Suppressor of cytokine signaling 2 is a feedback inhibitor of TLR-induced activation in human monocyte-derived dendritic cells. J Immunol 2011;187:2875-84.

13. Georgantas RW III, Tanadve V, Malehorn M, Heimfeld S, Chen C, Carr L, et al. Microarray and serial analysis of gene expression analyses identify known and novel transcripts overexpressed in hematopoietic stem cells. Cancer Res 2004;64:4434-41.

14. Ivanova NB, Dimos JT, Schaniel C, Hackney JA, Moore KA, Lemischka IR. A stem cell molecular signature. Science 2002;298:601-4.

15. Ramalho-Santos M, Yoon S, Matsuzaki Y, Mulligan RC, Melton DA "Stemness": transcriptional profiling of embryonic and adult stem cells. Science 2002;298:597-600.

16. Toren A, Bielorai B, Jacob-Hirsch J, Fisher T, Kreiser D, Moran O, et al. CD133-positive hematopoietic stem cell "stemness" genes contain many genes mutated or abnormally expressed in leukemia. Stem Cells 2005;23: 1142-53.

17. Kimura A, Martin C, Robinson GW, Simone JM, Chen W, Wickre MC, et al The gene encoding the hematopoietic stem cell regulator CCN3/NOV is under direct cytokine control through the transcription factors STAT5A/B. J Biol Chem 2010;285:32704-9.

18. Venezia TA, Merchant AA, Ramos CA, Whitehouse NL, Young AS, Shaw CA et al. Molecular signatures of proliferation and quiescence in hematopoietic stem cells. PLoS Biol 2004;2:e301.

19. Rico-Bautista E, Flores-Morales A, Fernandez-Perez L. Suppressor of cytokine signaling (SOCS) 2, a protein with multiple functions. Cytokine Growth Factor Rev 2006;17:431-9.

20. Dorritie KA, McCubrey JA, Johnson DE. STAT transcription factors in hematopoiesis and leukemogenesis: opportunities for therapeutic intervention. Leukemia 2014;28:248-57.

21. Randall TD, Weissman IL. Phenotypic and functional changes induced at the clonal level in hematopoietic stem cells after 5-fluorouracil treatment. Blood 1997;89:3596-606.

22. Zhong JF, Zhao Y, Sutton S, Su A, Zhan Y, Zhu L, et al. Gene expression profile of murine long-term reconstituting vs. short-term reconstituting hematopoietic stem cells. Proc Natl Acad Sci U S A 2005;102:2448-53.

23. Tripodo C, Sangaletti S, Guarnotta C, Piccaluga PP, Cacciatore M, Giuliano $\mathrm{M}$, et al. Stromal SPARC contributes to the detrimental fibrotic changes associated with myeloproliferation whereas its deficiency favors myeloid cell expansion. Blood 2012;120:3541-54.

24. De Palma M, Naldini L. Transduction of a gene expression cassette using advanced generation lentiviral vectors. Methods Enzymol 2002;346: 514-29.

25. Hansen N, Agerstam H, Wahlestedt M, Landberg N, Askmyr M, Ehinger M, et al. SOCS2 is dispensable for BCR/ABL1-induced chronic myeloid leukemia-like disease and for normal hematopoietic stem cell function. Leukemia 2013;27:130-5.
26. Kato $\mathrm{Y}$, Iwama A, Tadokoro $\mathrm{Y}$, Shimoda K, Minoguchi M, Akira S, et al. Selective activation of STAT5 unveils its role in stem cell self-renewal in normal and leukemic hematopoiesis. J Exp Med 2005;202:169-79.

27. Han L, Wierenga AT, Rozenveld-Geugien M, van de Lande K, Vellenga E, Schuringa JJ. Single-cell STAT5 signal transduction profiling in normal and leukemic stem and progenitor cell populations reveals highly distinct cytokine responses. PLoS ONE 2009;4:e7989.

28. Kalaitzidis D, Neel BG. Flow-cytometric phosphoprotein analysis reveals agonist and temporal differences in responses of murine hematopoietic stem/progenitor cells. PLoS ONE 2008;3:e3776.

29. Eppert K, Takenaka K, Lechman ER, Waldron L, Nilsson B, van Galen P, et al. Stem cell gene expression programs influence clinical outcome in human leukemia. Nat Med 2011;17:1086-93.

30. Wierenga AT, Vellenga E, Schuringa JJ. Maximal STAT5-induced proliferation and self-renewal at intermediate STAT5 activity levels. Mol Cell Biol 2008;28:6668-80.

31. Yoshida T, Ng SY, Zuniga-Pflucker JC, Georgopoulos K. Early hematopoietic lineage restrictions directed by Ikaros. Nat Immunol 2006;7:382-91.

32. Yagi $T$, Hibi $S$, Takanashi $M$, Kano G, Tabata $Y$, Imamura $T$, et al. High frequency of Ikaros isoform 6 expression in acute myelomonocytic and monocytic leukemias: implications for up-regulation of the antiapoptotic protein Bcl-XL in leukemogenesis. Blood 2002;99:1350-5.

33. Iacobucci I, Lonetti A, Messa F, Cilloni D, Arruga F, Ottaviani E, et al. Expression of spliced oncogenic Ikaros isoforms in Philadelphia-positive acute lymphoblastic leukemia patients treated with tyrosine kinase inhibitors: implications for a new mechanism of resistance. Blood 2008; 112:3847-55.

34. Nakayama H, Ishimaru F, Avitahl N, Sezaki N, Fujii N, Nakase K, et al Decreases in Ikaros activity correlate with blast crisis in patients with chronic myelogenous leukemia. Cancer Res 1999;59:3931-4.

35. Cante-Barrett K, Pieters R, Meijerink JP. Myocyte enhancer factor 2C in hematopoiesis and leukemia. Oncogene 2014;33:403-10.

36. Krivtsov AV, Twomey D, Feng Z, Stubbs MC, Wang Y, Faber J, et al. Transformation from committed progenitor to leukaemia stem cell initiated by MLL-AF9. Nature 2006;442:818-22

37. Margolin AA, Nemenman I, Basso K, Wiggins C, Stolovitzky G, Dalla Favera R, et al. ARACNE: an algorithm for the reconstruction of gene regulatory networks in a mammalian cellular context. BMC Bioinform 2006;7(Suppl 1):S7.

38. Metcalf D. Hematopoietic cytokines. Blood 2008;111:485-91.

39. Bradley HL, Hawley TS, Bunting KD. Cell intrinsic defects in cytokine responsiveness of STAT5-deficient hematopoietic stem cells. Blood 2002;100:3983-9

40. Frank O, Brors B, Fabarius A, Li L, Haak M, Merk S, et al. Gene expression signature of primary imatinib-resistant chronic myeloid leukemia patients. Leukemia 2006;20:1400-7.

41. Villuendas R, Steegmann JL, Pollan M, Tracey L, Granda A, Fernandez-Ruiz $\mathrm{E}$, et al. Identification of genes involved in imatinib resistance in CML: a gene-expression profiling approach. Leukemia. 2006;20:1047-54.

42. Schultheis B, Carapeti-Marootian M, Hochhaus A, Weisser A, Goldman JM, Melo JV. Overexpression of SOCS-2 in advanced stages of chronic myeloid leukemia: possible inadequacy of a negative feedback mechanism. Blood 2002;99:1766-75.

43. Kazi JU, Ronnstrand L. Suppressor of cytokine signaling 2 (SOCS2) associates with FLT3 and negatively regulates downstream signaling. Mol Oncol 2013;7:693-703

44. Iacobucci I, Lonetti A, Cilloni D, Messa F, Ferrari A, Zuntini R, et al. Identification of different Ikaros cDNA transcripts in Philadelphia-positive adult acute lymphoblastic leukemia by a high-throughput capillary electrophoresis sizing method. Haematologica 2008;93:1814-21.

45. Sun L, Crotty ML, Sensel M, Sather H, Navara C, Nachman J, et al. Expression of dominant-negative Ikaros isoforms in T-cell acute lymphoblastic leukemia. Clin Cancer Res 1999;5:2112-20.

46. Stehling-Sun S, Dade J, Nutt SL, DeKoter RP, Camargo FD. Regulation of lymphoid versus myeloid fate 'choice' by the transcription factor Mef2c. Nat Immunol 2009;10:289-96. 